\title{
Positivity and geometric function theory constraints on pion scattering
}

\author{
Ahmadullah Zahed \\ Centre for High Energy Physics, Indian Institute of Science, \\ C.V. Raman Avenue, Bangalore 560012, India \\ E-mail: ahmadullah@iisc.ac.in
}

ABSTRACT: This paper presents the fascinating correspondence between the geometric function theory and the scattering amplitudes with $O(N)$ global symmetry. A crucial ingredient to show such correspondence is a fully crossing symmetric dispersion relation in the $z$-variable, rather than the fixed channel dispersion relation. We have written down fully crossing symmetric dispersion relation for $O(N)$ model in $z$-variable for three independent combinations of isospin amplitudes. We have presented three independent sum rules or locality constraints for the $O(N)$ model arising from the fully crossing symmetric dispersion relations. We have derived three sets of positivity conditions. We have obtained two-sided bounds on Taylor coefficients of physical Pion amplitudes around the crossing symmetric point (for example, $\pi^{+} \pi^{-} \rightarrow \pi^{0} \pi^{0}$ ) applying the positivity conditions and the BieberbachRogosinski inequalities from geometric function theory.

KEywords: Effective Field Theories, Field Theories in Higher Dimensions, Nonperturbative Effects

ArXiv EPrint: 2108.10355 


\section{Contents}

1 Introduction 1

2 Crossing symmetric dispersion relation for $O(N)$ model 4

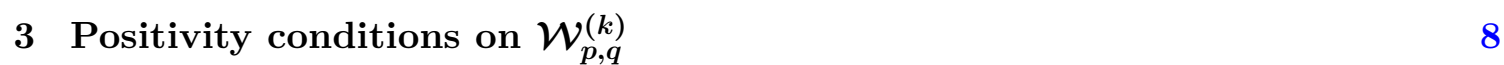

4 Geometric function theory for $O(N)$ model $\quad 10$

$\begin{array}{lll}4.1 \text { Typically real functions } & 10\end{array}$

$\begin{array}{ll}4.2 \text { Typically real functions for } O(N) \text { model } & 10\end{array}$

$\begin{array}{lll}4.3 & \text { Rogosinski bounds on } \alpha_{p}^{(k)}(a) & 12\end{array}$

5 Geometric function theory constraints on pion amplitudes 12

$\begin{array}{ll}5.1 \text { Bounds on coefficients } \widehat{\mathcal{W}}_{n-m, m}^{(k)} & 12\end{array}$

$\begin{array}{lll}5.2 & \text { Bounds on Taylor coefficients of physical amplitudes } & 15\end{array}$

$\begin{array}{lll}6 & \text { Summary and future directions } & 17\end{array}$

A Verification of various formulas against $O(3)$ Lovelace-Shapiro model $\quad 19$

$\begin{array}{ll}\text { A.1 Dispersion relation } & 19\end{array}$

$\begin{array}{lll}\text { A.2 Inversion formulas and sum rules } & 20\end{array}$

B Positivity conditions on of $\mathcal{W}_{n, m}^{(1)}, \mathcal{W}_{n, m}^{(2)} \quad 21$

B.1 Finding $\Upsilon_{n, r, m}^{(1)}(\mu, \delta), \Upsilon_{n, r, m}^{(2)}(\mu, \delta), \chi_{n, r, m}^{(1)}(\mu, \delta), \chi_{n, r, m}^{(2)}(\mu, \delta) \quad 21$

B.2 Proof of positivity conditions on of $\mathcal{W}_{n, m}^{(1)}, \mathcal{W}_{n, m}^{(2)} \quad 23$

C Positivity of Disc $\left[\left(1-\frac{2 \delta_{2, k}}{3}\right) \mathcal{G}_{0}+\delta_{1, k} \mathcal{G}_{1}+\delta_{2, k} \mathcal{G}_{2}\right]\left(s_{1}^{\prime} ; s_{2}^{(+)}\left(s_{1}^{\prime}, a\right)\right)$

D Verifications of bounds with known theories $\quad 24$

D.1 The 2-loop chiral perturbation theory 24

D.2 The $O(3)$ Lovelace-Shapiro model 25

\section{Introduction}

In geometric function theory, Bieberbach-Rogosinski inequalities tell us that the Taylor coefficients of typically real functions (also known as Herglotz function) are two-sided bounded $[1,2] \cdot{ }^{1}$ A typically real function $f(z)$, is a function that satisfies $\operatorname{Im}[f(z)] \operatorname{Im}[z]>$ $0, \operatorname{Im}[z] \neq 0$, i.e. the imaginary part of the function is positive in the upper half plane, and

\footnotetext{
${ }^{1}$ Translated version of [1] by P. Haldar, P. Raman and A. Zahed available on request.
} 
negative in the lower half plane. Let $f(z)$ be a typically real function with (normalized) Taylor expansion (inside the disk $|z|<1$ )

$$
f(z)=z+\sum_{p=2}^{\infty} b_{p} z^{p}
$$

Then the Bieberbach-Rogosinski inequalities say

$$
-\kappa_{p} \leq b_{p} \leq p
$$

where

$$
\kappa_{p}=-\operatorname{Min}\left(\frac{\sin p \theta}{\sin \theta}\right), 0 \leq \theta \leq \pi
$$

which is given by

$$
\kappa_{p}=-\frac{\sin p \varphi_{p}}{\sin \varphi_{p}}, \csc \left(\varphi_{p}\right)\left(p \cos \left(p \varphi_{p}\right)-\sin \left(p \varphi_{p}\right) \cot \left(\varphi_{p}\right)\right)=0
$$

For $p$ odd, $\pi / p<\varphi_{p}<3 \pi / 2 p$, while for $p$ even, $\varphi_{p}=\pi$ is only solution, giving $\kappa_{p}=p$.

For $p$ odd $\kappa_{p}$ given by solutions to the above equation [1-3]. For examples $\kappa_{3}=1, \kappa_{5} \approx$ $1.25, \kappa_{7} \approx 1.6, \ldots$

In [4] the correspondence between the famous Bieberbach conjecture (de Branges' theorem) and the non-perturbative crossing symmetric scattering amplitudes was pointed out, which established a close relationship between the Bieberbach-bounds and the bounds on the Wilson coefficients. In [3] it was pointed out that crossing symmetric scattering amplitudes are typically real functions. The pivotal ingredient in demonstrating such a correlation between the geometric function theory and the scattering amplitudes is crossing symmetric dispersion relation in a new $z$-variable for a fixed parameter $a$. The $z, a$ variables arise by parametrizing the Mandelstam invariants in the following way. The 2-2 scattering amplitudes with $O(N)$ global symmetry are functions of $s, t, u$, the Mandelstam invariants, satisfying $s+t+u=4 m^{2}$, where $m$ is the mass of the external scalars. For convenience, we will work with the notation $[5,6]$

$$
s_{1}=s-\frac{\mu}{3}, s_{2}=t-\frac{\mu}{3}, s_{3}=u-\frac{\mu}{3}=-s_{1}-s_{2}, \mu=4 m^{2} ;
$$

Fully crossing symmetric dispersion relation is written down by parametrizing $s_{i}$ as a function of $z, a$. The parametrization is given by

$$
s_{i}=a-\frac{a\left(z-z_{i}\right)^{3}}{z^{3}-1}, \quad i=1,2,3,
$$

where $a$ is a real parameter, $-\frac{\mu}{3} \leq a<\frac{2 \mu}{3}$ and $z_{i}$ is the cube roots of unity, after we parametrize $s_{i}$ as a function of $z, a$, the amplitude is a function of $z^{3}=\tilde{z}$, $a$, i.e. $\overline{\mathcal{M}}(\tilde{z}, a)$. The parameter $a$ is given by $a=\frac{y}{x}$, where $x=-\left(s_{1} s_{2}+s_{2} s_{3}+s_{3} s_{1}\right), y=-s_{1} s_{2} s_{3}$. For a crossing symmetric dispersion relation in $z$-variable, while keeping $a$-fixed $[5,6]$, the kernel is a univalent function, ${ }^{2}$ and the absorptive part is positive for a certain range of

\footnotetext{
${ }^{2} \mathrm{~A}$ function is univalent on a domain $\mathcal{D}$ if it is holomorphic, and one-to-one, i.e. for all $z_{1}, z_{2}$ in $\mathcal{D}$, $f\left(z_{1}\right)=f\left(z_{2}\right)$ if $z_{1}=z_{2}$.
} 
$a$. The kernel's specific form and the absorptive part's positivity enable one to re-express the dispersion relation as a Robertson representation. ${ }^{3}$ Once a function has a Robertson representation, we can say it is a typically real function. Therefore the amplitudes $\overline{\mathcal{M}}(\tilde{z}, a)$ are typically real functions. We ask here if such dispersion relation can be written for theories with $O(N)$ global symmetry? Do these correspondences exist for theories with global $O(N)$ symmetry? The answer turns out to be yes! We write down three sets of fully crossing symmetric dispersion relations for three specific combinations of isospin amplitudes. We call these combinations of isospin amplitudes to be $\mathcal{F}_{k}(\tilde{z}, a), \quad k=0,1,2$. We find that these combinations are in the Robertson representation. Hence we can write three sets of Bieberbach-Rogosinski inequalities. If we write

$$
\mathcal{F}_{k}(\tilde{z}, a)=\sum_{p=1}^{\infty} \alpha_{p}^{(k)}(a) a^{2 p} \tilde{z}^{p}, \quad k=0,1,2,
$$

the Bieberbach-Rogosinski inequalities take the form:

$$
-\kappa_{p} \leq \frac{\alpha_{p}^{(k)}(a) a^{2 p}}{\alpha_{1}^{(k)}(a) a^{2}} \leq p,
$$

for a range of $a$, which is derived in the main text (see eq (4.14)).

These three sets of dispersion relation and unitarity conditions give three sets of positivity constraints for Taylor coefficients of the amplitudes around the crossing symmetric point. These three sets of new positivity constraints are very non-trivial. Demanding the locality, we get three sets of independent sum rules or locality constraints. These novel sets of independent sum rules constrain the theories strongly.

There is a lot of recent work on constraining the quantum field theory using dispersion relation, utilizing the analyticity and unitarity assumptions [7-14]. Dispersion relations are the non-perturbative depiction of scattering amplitudes. Usually, people write dispersion relations in 2-2 scattering by keeping Mandelstam invariant $t$-fixed and write a dispersion integral in $s$-variable, which leads to the $s \leftrightarrow u$ symmetric representation of the amplitude. Imposing full crossing symmetry as an additional condition, one gets null constraints. Analogous strategies also developed in the context of conformal field theories, Mellin amplitudes. See for example [15-18].

The constraints in EFT Wilson coefficients were worked out in [19-31], using positivity of the partial wave and null conditions. ${ }^{4}$ In our case, we don't use the null constraints; instead, we use positivity (of the partial wave expansion) and bounds in the Taylor series coefficients of the amplitudes in $z$-variable that appear from the geometric function theory.

Bieberbach-Rogosinski inequalities and the positivity conditions give two-sided bounds on the Taylor coefficients of the amplitudes of any physical process around the crossing

\footnotetext{
${ }^{3}$ In $|z|<1$ a regular function $F(z)$ is typically real if and only if it has the Robertson representation:
}

$$
F(z)=\int_{-1}^{1} d f(\eta) \frac{z}{1-2 \eta z+z^{2}},
$$

where the measure $f(\eta)$ is a non-decreasing function.

${ }^{4}$ Locality constraints are the same as the null constraints. See [6] for more discussion. 
symmetric point ${ }^{5}$ these processes need not be fully crossing symmetric, only two channel symmetry is sufficient. For example, consider

$$
\mathcal{M}^{\left(\pi^{+} \pi^{-} \rightarrow \pi^{0} \pi^{0}\right)}\left(s_{1}, s_{2}\right)=\sum_{p=0, q=0}^{\infty} \mathcal{C}_{p, q}\left(-s_{2} s_{3}\right)^{p}\left(-s_{1}\right)^{q}
$$

This amplitude is not fully crossing symmetric. The three sets of BieberbachRogosinski inequalities and three sets of positivity conditions provide two-sided (order $\mathcal{O}(1)$, in [3] the $\mathcal{O}(1)$ is shown to be $|\mathcal{O}(1)|<5.625)$ bounds on the $\mathcal{C}_{p, q}$, presented in table 3.

We have organized the paper in the following way. The definitions of the fully crossing symmetric combinations, their dispersion relations, inversion formula and sum rules are presented in section 2. Section 3 contains positivity conditions on the Taylor coefficients of three crossing symmetric combinations. Section 4 describes how geometric function theory for the $O(N)$ model can be realized. Section 5 contains the application of the geometric function theory to physical pion amplitudes and bounds on Taylor coefficients of physical amplitudes around crossing symmetric point. We conclude with a summary and future directions in section 6 . We have added appendices for multiple demonstrations and verifications.

\section{Crossing symmetric dispersion relation for $O(N)$ model}

The 2-2 scattering amplitude with $O(N)$ global symmetry can be written as

$\mathcal{M}_{a b}^{c d}\left(s_{1}, s_{2}\right)=A\left(s_{1} \mid s_{2}, s_{3}\right) \delta_{a b} \delta^{c d}+A\left(s_{2} \mid s_{3}, s_{1}\right) \delta_{a}^{c} \delta_{b}^{d}+A\left(s_{3} \mid s_{1}, s_{2}\right) \delta_{a}^{d} \delta_{b}^{c}=\sum_{I=0}^{2} \mathcal{M}^{(I)}\left(s_{1}, s_{2}\right) \mathbb{P}_{I}$,

where $A\left(s_{i} \mid s_{j}, s_{k}\right)=A\left(s_{i} \mid s_{k}, s_{j}\right)$. The isospin $I$ s-channel amplitudes $\mathcal{M}^{(I)}\left(s_{1}, s_{2}\right)$ are given by

$$
\begin{aligned}
& \mathcal{M}^{(0)}\left(s_{1}, s_{2}\right)=N A\left(s_{1} \mid s_{2}, s_{3}\right)+A\left(s_{2} \mid s_{3}, s_{1}\right)+A\left(s_{3} \mid s_{1}, s_{2}\right) \\
& \mathcal{M}^{(1)}\left(s_{1}, s_{2}\right)=A\left(s_{2} \mid s_{3}, s_{1}\right)-A\left(s_{3} \mid s_{1}, s_{2}\right) \\
& \mathcal{M}^{(2)}\left(s_{1}, s_{2}\right)=A\left(s_{2} \mid s_{3}, s_{1}\right)+A\left(s_{3} \mid s_{1}, s_{2}\right)
\end{aligned}
$$

with

$$
\mathbb{P}_{0}=\frac{1}{N} \delta_{a b} \delta^{c d}, \quad \mathbb{P}_{1}=\frac{1}{2}\left(\delta_{a}^{c} \delta_{b}^{d}-\delta_{a}^{d} \delta_{b}^{c}\right), \quad \mathbb{P}_{2}=\frac{1}{2}\left(\delta_{a}^{c} \delta_{b}^{d}+\delta_{a}^{d} \delta_{b}^{c}-\frac{2}{N} \delta_{a b} \delta^{\mathrm{cd}}\right) .
$$

We want to write down crossing symmetric dispersion relation in $z$-variable for fixed $a$ following [5,6] for $O(N)$ model. We are interested in such a crossing symmetric dispersion relation to connecting with the geometric function theory. In general full 3-channel crossing

\footnotetext{
${ }^{5}$ The Bieberbach-Rogosinski inequalities and the positivity conditions are insufficient to put two-sided bounds for solely two-channel symmetric amplitudes without global symmetry. There non-linear inequalities and null constraints are needed. See [3] for further details. We thank Prashanth Raman for pointing out this to us.
} 
symmetry is missing in isospin amplitudes $\mathcal{M}^{(I)}\left(s_{1}, s_{2}\right)$. Following [32, 33], we will consider independent fully crossing symmetric combinations

$$
\begin{aligned}
\mathcal{G}_{0}\left(s_{1}, s_{2}\right)= & \frac{\mathcal{M}^{(0)}\left(s_{1}, s_{2}\right)}{N}+\frac{(N-1) \mathcal{M}^{(2)}\left(s_{1}, s_{2}\right)}{N}, \\
\mathcal{G}_{1}\left(s_{1}, s_{2}\right)= & \frac{\mathcal{M}^{(1)}\left(s_{3}, s_{1}\right)}{s_{1}-s_{2}}+\frac{\mathcal{M}^{(1)}\left(s_{1}, s_{2}\right)}{s_{2}-s_{3}}+\frac{\mathcal{M}^{(1)}\left(s_{2}, s_{3}\right)}{s_{3}-s_{1}} \\
\mathcal{G}_{2}\left(s_{1}, s_{2}\right)= & \frac{\frac{\mathcal{M}^{(1)}\left(s_{1}, s_{2}\right)}{s_{2}-s_{3}}+\frac{\mathcal{M}^{(1)}\left(s_{2}, s_{1}\right)}{s_{3}-s_{1}}}{s_{1}-s_{2}}+\frac{\frac{\mathcal{M}^{(1)}\left(s_{2}, s_{3}\right)}{s_{3}-s_{1}}+\frac{\mathcal{M}^{(1)}\left(s_{3}, s_{2}\right)}{s_{1}-s_{2}}}{s_{2}-s_{3}} \\
& +\frac{\frac{\mathcal{M}^{(1)}\left(s_{1}, s_{3}\right)}{s_{2}-s_{3}}+\frac{\mathcal{M}^{(1)}\left(s_{3}, s_{1}\right)}{s_{1}-s_{2}}}{s_{3}-s_{1}}
\end{aligned}
$$

The $\mathcal{G}_{0}\left(s_{1}, s_{2}\right)$ is the $\pi^{0} \pi^{0} \rightarrow \pi^{0} \pi^{0}$ amplitude. Dispersion relation in $z$-variable for $\mathcal{G}_{0}\left(s_{1}, s_{2}\right)$ is discussed in $[5,6]$, and relations to geometric function theory is given in detail in $[3,4]$. We will write $z$-variable dispersion relations for all three of the $\mathcal{G}_{k}\left(s_{1}, s_{2}\right)$ and demonstrate that $\mathcal{G}_{k}\left(s_{1}, s_{2}\right)$ (linear combinations of them) are related to geometric function theory. We expect that $\mathcal{G}_{k}\left(s_{1}, s_{2}\right)$ to have the same analyticity properties as the isospin amplitude $\mathcal{M}^{(I)}\left(s_{1}, s_{2}\right)$. Since antisymmetry of $\mathcal{M}^{(1)}\left(s_{1}, s_{2}\right)$ with respect to $s_{2}$ and $s_{3}$, prevents the denominators appearing in $\mathcal{G}_{1}\left(s_{1}, s_{2}\right)$ and $\mathcal{G}_{2}\left(s_{1}, s_{2}\right)$ to introduce any additional new singularities at $s_{1}=$ $s_{2}, s_{2}=s_{3}, s_{3}=s_{1}$. From the large $s_{1}$, fixed $s_{2}$ behaviour of the isospin amplitudes $\mathcal{M}^{(I)}\left(s_{1}, s_{2}\right)$, we can write once ${ }^{6}$ subtracted dispersion relation in $z$-variable for fixed $a$ for $\mathcal{G}_{k}\left(s_{1}, s_{2}\right)$. For large $s_{1}$ fixed $s_{2}$ we have

$$
\mathcal{G}_{k}\left(s_{1}, s_{2}\right)=o\left(s_{1}^{2}\right) \text {, for fixed } s_{2} .
$$

The discontinuity of $\mathcal{G}_{k}\left(s_{1}, s_{2}\right)$ starts at $s_{1}=\frac{2 \mu}{3}$. Therefore, once we know the discontinuity of $\mathcal{G}_{k}\left(s_{1}, s_{2}\right)$, we can write fully crossing symmetric dispersion relations, following the same logic in $[5,6]$. This leads to fully crossing symmetric dispersion relation ${ }^{7}$ is $s_{1}, s_{2}$ variables

$$
\mathcal{G}_{k}\left(s_{1}, s_{2}\right)=\alpha_{0}^{(k)}+\frac{1}{\pi} \int_{\frac{2 \mu}{3}}^{\infty} \frac{d s_{1}^{\prime}}{s_{1}^{\prime}} \operatorname{Disc} \mathcal{G}_{k}\left(s_{1}^{\prime} ; s_{2}^{(+)}\left(s_{1}^{\prime}, a\right)\right) H\left(s_{1}^{\prime} ; s_{1}, s_{2}, s_{3}\right) ; k=0,1,2
$$

where $\alpha_{0}^{(k)}$ is the subtraction constant. The $\operatorname{Disc} \mathcal{G}_{k}\left(s_{1} ; s_{2}\right)$ is the $s$-channel discontinuity of $\mathcal{G}_{k}\left(s_{1}, s_{2}\right)$ and

$$
\begin{aligned}
H\left(s_{1}^{\prime} ; s_{1}, s_{2}, s_{3}\right) & =\left[s_{1}\left(s_{1}^{\prime}-s_{1}\right)^{-1}+s_{2}\left(s_{1}^{\prime}-s_{2}\right)^{-1}+s_{3}\left(s_{1}^{\prime}-s_{3}\right)^{-1}\right], \\
s_{2}^{(+)}\left(s_{1}^{\prime}, a\right) & =-\frac{s_{1}^{\prime}}{2}\left[1-\left(\frac{s_{1}^{\prime}+3 a}{s_{1}^{\prime}-a}\right)^{1 / 2}\right], a=\frac{s_{1} s_{2} s_{3}}{s_{1} s_{2}+s_{2} s_{3}+s_{3} s_{1}} .
\end{aligned}
$$

Partial wave expansion for $\mathcal{G}_{k}\left(s_{1}, s_{2}\right)$ and it's convergence has been discussed in [32] (see [34] for some applications). Since the $\mathcal{G}_{k}\left(s_{1}, s_{2}\right)$ have the same analyticity properties as the

\footnotetext{
${ }^{6}$ For $\mathcal{G}_{2}\left(s_{1}, s_{2}\right)$ we can write a dispersion relation without subtraction, which we will not use here.

${ }^{7}$ Crossing symmetric dispersion relation in [32] are in $x, y$ variables with a complicated kernel, not in $z$-variable. It is not clear if, from the dispersion relation in [32], one can connect with geometric function theory.
} 
isospin amplitude $\mathcal{M}^{(I)}\left(s_{1}, s_{2}\right)$ and $\mathcal{M}^{(I)}\left(s_{1}, s_{2}\right)$ has cuts starting at $s_{1} \geq 2 \mu / 3$, hence the domain of $a$ is same as discussed in [5]. The range of $a$, we will consider here is $-\frac{\mu}{3} \leq a<\frac{2 \mu}{3}$. This range of $a$ can be enlarged to $-6.71 \mu \leq a<\frac{2 \mu}{3}$ as discussed in [5, see appendix ], [32, see discussion below eq. 3.10]. We will use that in our calculations in the upcoming sections.

Below we have presented the formula for $s$-channel discontinuity, $\operatorname{Disc} \mathcal{G}_{k}\left(s_{1} ; s_{2}\right)$ :

$\operatorname{Disc} \mathcal{G}_{0}\left(s_{1} ; s_{2}\right)=\frac{\mathcal{A}^{(0)}\left(s_{1}, s_{2}\right)+(N-1) \mathcal{A}^{(2)}\left(s_{1}, s_{2}\right)}{N}$,

$\operatorname{Disc} \mathcal{G}_{1}\left(s_{1} ; s_{2}\right)=\frac{3 s_{1}\left(s_{1}+2 s_{2}\right)\left(2 \mathcal{A}^{(0)}\left(s_{1}, s_{2}\right)-(N+2)\right) \mathcal{A}^{(2)}\left(s_{1}, s_{2}\right)+3 N\left(s_{1}^{2}-2 s_{2} s_{1}-2 s_{2}^{2}\right) \mathcal{A}^{(1)}\left(s_{1}, s_{2}\right)}{2 N\left(s_{1}-s_{2}\right)\left(2 s_{1}+s_{2}\right)\left(s_{1}+2 s_{2}\right)}$,

$\operatorname{Disc}_{\mathcal{G}_{2}}\left(s_{1} ; s_{2}\right)=\frac{3\left(\left(s_{1}+2 s_{2}\right)\left(-2 \mathcal{A}^{(0)}\left(s_{1}, s_{2}\right)+(N+2)\right) \mathcal{A}^{(2)}\left(s_{1}, s_{2}\right)+3 N s_{1} \mathcal{A}^{(1)}\left(s_{1}, s_{2}\right)\right)}{2 N\left(s_{1}-s_{2}\right)\left(2 s_{1}+s_{2}\right)\left(s_{1}+2 s_{2}\right)}$,

where, $\mathcal{A}^{(I)}\left(s_{1}, s_{2}\right)$ is the $s$-channel discontinuity of the isospin amplitude $\mathcal{M}^{(I)}\left(s_{1}, s_{2}\right)$. The $s$-channel discontinuity of the isospin amplitude, $\mathcal{A}^{(I)}\left(s_{1}, s_{2}\right)$ has a partial wave expansion

$$
\begin{aligned}
\mathcal{A}^{(I)}\left(s_{1}, s_{2}^{(+)}\left(s_{1}, a\right)\right) & =\Phi\left(s_{1} ; \alpha\right) \sum_{\ell=0}^{\infty}(2 \ell+2 \alpha) a_{\ell}^{(I)}\left(s_{1}\right) C_{\ell}^{(\alpha)}\left(\sqrt{\xi\left(s_{1}, a\right)}\right) \\
\xi\left(s_{1}, a\right) & =\cos ^{2} \theta_{s}=\left(1+\frac{2 s_{2}^{+}\left(s_{1}, a\right)+\frac{2 \mu}{3}}{s_{1}-\frac{2 \mu}{3}}\right)^{2}=\xi_{0}+4 \xi_{0}\left(\frac{a}{s_{1}-a}\right) .
\end{aligned}
$$

We normalize $C_{\ell}^{(\alpha)}$ such that $\Phi\left(s_{1}, \alpha\right)=\Psi(\alpha) \frac{\sqrt{s_{1}+\frac{\mu}{3}}}{\left(s_{1}-\frac{2 \mu}{3}\right)^{\alpha}}$ with $\Psi(\alpha)>0$ i.e $\Psi(\alpha)$ is a real positive number. More importantly, due to unitarity, the partial wave coefficients satisfy

$$
0 \leq a_{\ell}^{(I)}\left(s_{1}\right) \leq 1
$$

For our calculations, we will be only utilizing the positivity of the partial wave coefficients. The isospin amplitudes can be written in terms of $\mathcal{G}_{k}\left(s_{1}, s_{2}\right)$ in the following way

$$
\begin{aligned}
\mathcal{M}^{(0)}\left(s_{1}, s_{2}\right) & =\frac{1}{9}\left(3(N+2) \mathcal{G}_{0}\left(s_{1}, s_{2}\right)+(N-1)\left(3 s_{1} \mathcal{G}_{1}\left(s_{1}, s_{2}\right)+\left(-s_{1}^{2}+2 s_{2} s_{1}+2 s_{2}^{2}\right) \mathcal{G}_{2}\left(s_{1}, s_{2}\right)\right)\right), \\
\mathcal{M}^{(1)}\left(s_{1}, s_{2}\right) & =\frac{1}{3}\left(s_{1}+2 s_{2}\right)\left(\mathcal{G}_{1}\left(s_{1}, s_{2}\right)+s_{1} \mathcal{G}_{2}\left(s_{1}, s_{2}\right)\right), \\
\mathcal{M}^{(2)}\left(s_{1}, s_{2}\right) & =\frac{1}{9}\left(6 \mathcal{G}_{0}\left(s_{1}, s_{2}\right)-3 s_{1} \mathcal{G}_{1}\left(s_{1}, s_{2}\right)+\left(s_{1}^{2}-2 s_{2} s_{1}-2 s_{2}^{2}\right) \mathcal{G}_{2}\left(s_{1}, s_{2}\right)\right) .
\end{aligned}
$$

These three equations are crucial. Once the Taylor coefficients of the expansion of $\mathcal{G}_{k}\left(s_{1}, s_{2}\right)$ are bounded (see equation (2.14) and table 2), utilizing the above three formulae (2.13), we can bound the Taylor expansion (around crossing symmetric point) coefficients of amplitude of any physical process.

Inversion formulas and sum rules. The $\mathcal{G}_{k}\left(s_{1}, s_{2}\right)$ have the same analyticity properties as the isospin amplitude $\mathcal{M}^{(I)}\left(s_{1}, s_{2}\right)$, and they don't have any additional singularities. More importantly, all three of them are fully crossing symmetric. Therefore, we can write

$$
\mathcal{G}_{k}\left(s_{1}, s_{2}\right)=\sum_{p, q=0}^{\infty} \mathcal{W}_{p q}^{(k)} x^{p} y^{q} ; k=0,1,2
$$


with crossing symmetric variables $x=-\left(s_{1} s_{2}+s_{2} s_{3}+s_{3} s_{1}\right), y=-s_{1} s_{2} s_{3}$. In the $z$ variable the kernel takes the form $H\left(s_{1}^{\prime} ; s_{1}, s_{2}, s_{3}\right)=\frac{27 a^{2} z^{3}\left(3 a-2 s_{1}^{\prime}\right)}{-27 a^{3} z^{3}+27 a^{2} z^{3} s_{1}^{\prime}+\left(z^{3}-1\right)^{2}\left(s_{1}^{\prime}\right)^{3}}$, which can be seen by writing $s_{i}$ 's in terms of $(z, a)$. Now identifying crossing symmetric variable $x$ in terms of $z$-variable via the relation $\frac{z^{3}}{\left(z^{3}-1\right)^{2}}=\frac{-x}{27 a^{2}}$, we can series expand in powers of $x$. We obtain

$$
\mathcal{G}_{k}\left(s_{1}, s_{2}\right)=\alpha_{0}^{(k)}+\sum_{n=1}^{\infty} \frac{1}{\pi} \int_{2 \mu / 3}^{\infty} d s_{1}^{\prime} \frac{\operatorname{Disc} \mathcal{G}_{k}\left(s_{1}^{\prime} ; s_{2}^{(+)}\left(s_{1}^{\prime}, a\right)\right)}{s_{1}^{\prime 2 n+1}}\left(1-\frac{a}{s_{1}^{\prime}}\right)^{n-1}\left(2-\frac{3 a}{s_{1}^{\prime}}\right) x^{n}
$$

Coefficient of $a^{m}($ since $a=y / x)$ will be $\mathcal{W}_{n-m, m}$. In general, one can write

$$
\mathcal{W}_{n-m, m}^{(k)}=\int_{2 \mu / 3}^{\infty} \frac{d s_{1}}{s_{1}} \Phi\left(s_{1} ; \alpha\right) \sum_{\ell=0}^{\infty}(2 \ell+2 \alpha) \mathcal{B}_{n, m, \ell}^{(k)}\left(s_{1}\right), n \geq 1
$$

The Disc $\mathcal{G}_{k}$ are given in terms of the partial wave coefficients of the $s$-channel discontinuity of the isospin amplitudes,

$\operatorname{Disc} \mathcal{G}_{0}\left(s_{1}, s_{2}^{(+)}\left(s_{1}, a\right)\right)=\Phi\left(s_{1} ; \alpha\right) \sum_{\ell=0}^{\infty}(2 \ell+2 \alpha)\left(\frac{a_{\ell}^{(0)}\left(s_{1}\right)}{N}+\frac{(N-1) a_{\ell}^{(2)}\left(s_{1}\right)}{N}\right) C_{\ell}^{(\alpha)}\left(\sqrt{\xi\left(s_{1}, a\right)}\right)$

$\operatorname{Disc}_{1}\left(s_{1}, s_{2}^{(+)}\left(s_{1}, a\right)\right)=\Phi\left(s_{1} ; \alpha\right) \sum_{\ell=0}^{\infty}(2 \ell+2 \alpha) 3\left(\frac{\left(s_{1}-a\right) a_{\ell}^{(0)}\left(s_{1}\right)}{N s_{1}\left(2 s_{1}-3 a\right)}+\frac{\sqrt{s_{1}-a}\left(s_{1}-3 a\right) a_{\ell}^{(1)}\left(s_{1}\right)}{2 s_{1} \sqrt{3 a+s_{1}}\left(2 s_{1}-3 a\right)}\right.$

$$
\left.+\frac{(N+2)\left(a-s_{1}\right) a_{\ell}^{(2)}\left(s_{1}\right)}{2 N s_{1}\left(2 s_{1}-3 a\right)}\right) C_{\ell}^{(\alpha)}\left(\sqrt{\xi\left(s_{1}, a\right)}\right)
$$

$\operatorname{Disc}_{2}\left(s_{1}, s_{2}^{(+)}\left(s_{1}, a\right)\right)=\Phi\left(s_{1} ; \alpha\right) \sum_{\ell=0}^{\infty}(2 \ell+2 \alpha) 3\left(a-s_{1}\right)\left(\frac{(N+2) a_{\ell}^{(2)}\left(s_{1}\right)-2 a_{\ell}^{(0)}\left(s_{1}\right)}{2 N s_{1}^{2}\left(3 a-2 s_{1}\right)}\right.$

$$
\left.+\frac{3 \sqrt{s_{1}-a} a_{\ell}^{(1)}\left(s_{1}\right)}{2 s_{1}^{2}\left(3 a-2 s_{1}\right) \sqrt{3 a+s_{1}}}\right) C_{\ell}^{(\alpha)}\left(\sqrt{\xi\left(s_{1}, a\right)}\right) \text {. }
$$

We use the same convention as [6]. The $\alpha=\frac{d-3}{2}, \Phi\left(s_{1} ; \alpha\right)=\Psi(\alpha) \frac{\sqrt{s_{1}+\frac{\mu}{3}}}{\left(s_{1}-\frac{2 \mu}{3}\right)^{\alpha}}$ where $\Psi(\alpha)>0$ is real and $\xi\left(s_{1}, a\right)=\xi_{0}+4 \xi_{0}\left(\frac{a}{s_{1}-a}\right), \quad \xi_{0}=\frac{s_{1}^{2}}{\left(s_{1}-2 \mu / 3\right)^{2}}$. The Gegenbauer polynomials can be expanded as $C_{\ell}^{(\alpha)}\left(\xi^{1 / 2}\right)=\sum_{j=0}^{\ell / 2} \frac{p_{\ell}^{(j)}\left(\xi_{0}\right)}{j !}\left(\xi-\xi_{0}\right)^{j}$ with $p_{\ell}^{(j)}\left(\xi_{0}\right)=\left.\frac{\partial^{j} C_{\ell}^{(\alpha)}(\sqrt{\xi})}{\partial \xi^{j}}\right|_{\xi=\xi_{0}}$. Now we plug the formulas (2.17) for $\operatorname{Disc} \mathcal{G}_{k}$ in the equation (2.15). After that we compute the coefficient of $a^{m}$. The coefficient of $a^{m}$ gives us formula

$$
\mathcal{B}_{n, m, \ell}^{(0)}\left(s_{1}\right)=\left(\frac{a_{\ell}^{(0)}\left(s_{1}\right)}{N}+\frac{(N-1) a_{\ell}^{(2)}\left(s_{1}\right)}{N}\right) \frac{1}{\pi} \sum_{j=0}^{m} \frac{1}{s_{1}^{2 n+m}} \frac{p_{\ell}^{(j)}\left(\xi_{0}\right)}{j !}\left(4 \xi_{0}\right)^{j} \times \frac{(3 j-m-2 n)(-n)_{m}}{(m-j) !(-n)_{j+1}} .
$$


Similarly

$$
\begin{aligned}
\mathcal{B}_{n, m, \ell}^{(1)}\left(s_{1}\right)= & \frac{1}{\pi} \sum_{j=0}^{m} \frac{1}{s_{1}^{2 n+m+1}} \frac{p_{\ell}^{(j)}\left(\xi_{0}\right)}{j !}\left(4 \xi_{0}\right)^{j} \times \frac{3}{2 N}\left[(-1)^{m-j}\left(\begin{array}{c}
n-j \\
m-j
\end{array}\right)\left(2 a_{\ell}^{(0)}\left(s_{1}\right)-(N+2) a_{\ell}^{(2)}\left(s_{1}\right)\right)\right. \\
& +N 3^{m-j} a_{\ell}^{(1)}\left(s_{1}\right)\left\{\left(\begin{array}{c}
-\frac{1}{2} \\
m-j
\end{array}\right){ }_{2} F_{1}\left(j-m, j-n+\frac{1}{2} ; j-m+\frac{1}{2} ;-\frac{1}{3}\right)\right. \\
& \left.\left.-\left(\begin{array}{c}
-\frac{1}{2} \\
-j+m-1
\end{array}\right){ }_{2} F_{1}\left(j-m+1, j-n+\frac{1}{2} ; j-m+\frac{3}{2} ;-\frac{1}{3}\right)\right\}\right]
\end{aligned}
$$

and

$$
\begin{aligned}
\mathcal{B}_{n, m, \ell}^{(2)}\left(s_{1}\right)= & \frac{1}{\pi} \sum_{j=0}^{m} \frac{1}{s_{1}^{2 n+m+2}} \frac{p_{\ell}^{(j)}\left(\xi_{0}\right)}{j !}\left(4 \xi_{0}\right)^{j} \times \frac{3}{N}\left[(-1)^{m-j}\left(\begin{array}{c}
n-j \\
m-j
\end{array}\right)\left((N+2) a_{\ell}^{(2)}\left(s_{1}\right)-2 a_{\ell}^{(0)}\left(s_{1}\right)\right)\right. \\
& \left.+N 3^{-j+m+1}\left(\begin{array}{c}
-\frac{1}{2} \\
m-j
\end{array}\right) a_{\ell}^{(1)}\left(s_{1}\right)_{2} F_{1}\left(j-m, j-n-\frac{1}{2} ; j-m+\frac{1}{2} ;-\frac{1}{3}\right)\right] .
\end{aligned}
$$

In the appendix, we have verified the dispersion relation and inversion formula against $\mathrm{O}(3)$ Lovelace-Shapiro model, see appendix A.

Sum rules. In the equation (2.14), only positive powers of $x, y$ appears. Our dispersion relation is fully crossing symmetric by construction. After writing down the dispersion relations, there appear to be negative powers of $x$ in the expansion. Coefficients of such terms are $\mathcal{W}_{n-m, m}^{(k)}, m>n$. These terms $\mathcal{W}_{n-m, m}^{(k)}, m>n$ does not vanish on their own. Negative powers of $x, y$, are non-local terms. Since there should not be any non-local terms, we should have

$$
\mathcal{W}_{n-m, m}^{(k)}=0, \text { for } m>n, k=0,1,2 .
$$

These conditions put very non-trivial constraints on the partial wave coefficients. We call these sum rules locality constraints. In the appendix, we have verified the sum rules against $\mathrm{O}(3)$ Lovelace-Shapiro model, see appendix A.

\section{Positivity conditions on $\mathcal{W}_{p, q}^{(k)}$}

In [6] the positivity conditions on $\mathcal{W}_{p, q}^{(0)}$ were derived, using the positivity of the partial wave coefficients and the positivity of Gegenbauer polynomials,

$$
\begin{aligned}
& \sum_{r=0}^{m} \chi_{n, r, m}^{(0)}\left(\mu, \delta_{0}\right) \mathcal{W}_{n-r, r}^{(0)} \geq 0 \\
& 0 \leq \mathcal{W}_{n, 0}^{(0)} \leq \frac{1}{\left(\delta_{0}+\frac{2 \mu}{3}\right)^{2}} \mathcal{W}_{n-1,0}^{(0)}, \quad n \geq 2
\end{aligned}
$$


Where $\delta_{0}$ is the scale ${ }^{8}$ of the theory and

$$
\begin{aligned}
\chi_{n, m, m}^{(0)}(\mu, \delta) & =1, \chi_{n, r, m}^{(0)}(\mu, \delta)=\sum_{j=r+1}^{m}(-1)^{j+r+1} \chi_{n, j, m}^{(0)} \frac{\mathfrak{U}^{\alpha}{ }_{n, j, r}\left(\delta+\frac{2 \mu}{3}\right)}{\mathfrak{U}^{\alpha}{ }_{n, r, r}\left(\delta+\frac{2 \mu}{3}\right)}, \\
\mathfrak{U}_{n, m, k}^{\alpha} & =\sum_{k=0}^{m} \frac{{\sqrt{16 \xi_{0}}}^{k}(\alpha)_{k}(m+2 n-3 j) \Gamma(n-j) \Gamma(2 j-k)}{s_{1}^{m+2 n} \Gamma(k) j !(m-j) !(j-k) !(n-m) !} .
\end{aligned}
$$

There exist similar kinds of (but very non-trivial) positivity conditions for $\mathcal{W}_{p, q}^{(1)}$ and $\mathcal{W}_{p, q}^{(2)}$, which take the following form

$$
\sum_{r=0}^{m}\left(\Upsilon_{n, r, m}^{(1)}\left(\mu, \delta_{0}\right) \mathcal{W}_{n-r, r}^{(1)}+\chi_{n, r, m}^{(1)}\left(\mu, \delta_{0}\right) \mathcal{W}_{n-r, r}^{(0)}\right) \geq 0
$$

and

$$
\sum_{r=0}^{m}\left(\Upsilon_{n, r, m}^{(2)}\left(\mu, \delta_{0}\right) \mathcal{W}_{n-r, r}^{(2)}+\chi_{n, r, m}^{(2)}\left(\mu, \delta_{0}\right) \mathcal{W}_{n-r, r}^{(0)}\right) \geq 0
$$

with $\Upsilon_{n, m, m}^{(1)}\left(\mu, \delta_{0}\right)=1, \Upsilon_{n, m, m}^{(2)}\left(\mu, \delta_{0}\right)=1$.

The $\chi_{n, r, m}^{(1)}\left(\mu, \delta_{0}\right), \chi_{n, r, m}^{(2)}\left(\mu, \delta_{0}\right), \Upsilon_{n, r, m}^{(1)}\left(\mu, \delta_{0}\right), \Upsilon_{n, r, m}^{(2)}\left(\mu, \delta_{0}\right)$ are known positive coefficients. Its quite hard to get a general formula, but one can work out case by case in $m$. For example, below, we listed them up to $m=3$.

$$
\begin{array}{|l|l|l|}
\hline \chi_{n, 0,1}^{(1)}(\delta, \mu)=\frac{27(2 n+9)(N+2)}{8(N-1)(3 \delta+2 \mu)^{2}} & \Upsilon_{n, 0,1}^{(1)}(\delta, \mu)=\frac{3(n+4)}{3 \delta+2 \mu} & \chi_{n, 1,1}^{(1)}(\delta, \mu)=\frac{9(N+2)}{4(N-1)(3 \delta+2 \mu)} \\
\hline \chi_{n, 0,2}^{(1)}(\delta, \mu)=\frac{81(2 n(n+1) 0+51)(N+2)}{16(N-1)(3 \delta+2 \mu)^{3}} & \chi_{n, 1,2}^{(1)}(\delta, \mu)=\frac{27(2 n+9)(N+2)}{8(N-1)(3 \delta+2 \mu)^{2}} & \Upsilon_{n, 0,2}^{(1)}(\delta, \mu)=\frac{9(n+4)(n+5)}{2(3 \delta+2 \mu)^{2}} \\
\hline \Upsilon_{n, 1,2}^{(1)}(\delta, \mu)=\frac{3(n+4)}{3 \delta+2 \mu} & \chi_{n, 2,2}^{(1)}(\delta, \mu)=\frac{99(N+2)}{4(N-1)(3 \delta+2 \mu)} & \chi_{n, 0,3}^{(1)}(\delta, \mu)=\frac{81(2 n+(n(2 n+33)+172)+879)(N+2)}{32(N-1)(3 \delta+2 \mu)} \\
\hline \chi_{n, 1,3}^{(1)}(\delta, \mu)=\frac{81(2 n(n+10)+47)(N+2)}{16(N-1)(3 \delta+2 \mu)^{3}} & \chi_{n, 2,3}^{(1)}(\delta, \mu)=\frac{27(2 n+9)(N+2)}{8(N-1)(3 \delta+2 \mu)^{2}} & \Upsilon_{n, 0,3}^{(1)}(\delta, \mu)=\frac{9(n(n(n)+15)+68)+168)}{2(3 \delta+2 \mu)^{3}} \\
\hline \Upsilon_{n, 1,3}^{(1)}(\delta, \mu)=\frac{9(n+3)(n+6)}{2(3 \delta+2 \mu)^{2}} & \Upsilon_{n, 2,3}^{(1)}(\delta, \mu)=\frac{3(n+4)}{3 \delta+2 \mu} & \chi_{n, 3,3}^{(1)}(\delta, \mu)=\frac{9(N+2)}{4(N-1)(3 \delta+2 \mu)} \\
\hline
\end{array}
$$

and

$$
\begin{array}{|l|l|l|}
\hline \chi_{n, 0,1}^{(2)}(\delta, \mu)=\frac{81(2 n+5)}{4(3 \delta+2 \mu)^{3}} & \Upsilon_{n, 0,1}^{(2)}=\frac{3(n+2)}{3 \delta+2 \mu} & \chi_{n, 1,1}^{(2)}(\delta, \mu)=\frac{27}{2(3 \delta+2 \mu)^{2}} \\
\hline \chi_{n, 0,2}^{(2)}(\delta, \mu)=\frac{243(2 n(n+6)+7)}{8(3 \delta+2 \mu)^{4}} & \chi_{n, 1,2}^{(2)}(\delta, \mu)=\frac{81(2 n+5)}{4(3 \delta+2 \mu)^{3}} & \Upsilon_{n, 0,2}^{(2)}(\delta, \mu)=\frac{9 n(n+5)}{2(3 \delta+2 \mu)^{2}} \\
\hline \Upsilon_{n, 1,2}^{(2)}(\delta, \mu)=\frac{3(n+2)}{3 \delta+2 \mu} & \chi_{n, 2,2}^{(2)}(\delta, \mu)=\frac{27}{2(3 \delta+2 \mu)^{2}} & \chi_{n, 0,3}^{(2)}(\delta, \mu)=\frac{243(2 n(n(2 n+21)+28)+51)}{16(3 \delta+2 \mu)^{5}} \\
\hline \chi_{n, 1,3}^{(2)}(\delta, \mu)=\frac{243(2 n(n+6)+3)}{8(3 \delta+2 \mu)^{4}} & \chi_{n, 2,3}^{(2)}(\delta, \mu)=\frac{81(2 n+5)}{4(3 \delta+2 \mu)^{3}} & \Upsilon_{n, 0,3}^{(2)}(\delta, \mu)=\frac{9 n(n(n+9)+2)}{2(3 \delta+2 \mu)^{3}} \\
\hline \Upsilon_{n, 1,3}^{(2)}(\delta, \mu)=\frac{9(n(n+5)-2)}{2(3 \delta+2 \mu)^{2}} & \Upsilon_{n, 2,3}^{(2)}(\delta, \mu)=\frac{3(n+2)}{3 \delta+2 \mu} & \chi_{n, 3,3}^{(2)}(\delta, \mu)=\frac{27}{2(3 \delta+2 \mu)^{2}} \\
\hline
\end{array}
$$

These positivity conditions follow from the positivity of partial wave coefficients $a_{\ell}^{(I)}\left(s_{1}\right)$ presented in the equation (2.12) and the positivity of Gegenbauer polynomials. Proof and derivation of these positivity conditions can be found in appendix B.

\footnotetext{
${ }^{8}$ For EFTs the analysis is modified by taking the lower limit of the integral $\frac{2 \mu}{3} \rightarrow \frac{2 \mu}{3}+\delta_{0}$, here $\delta_{0}$ will be the EFT scale. Since for EFTs, most of the time the low energy amplitude DiscG $\left.\right|_{\frac{2 \mu}{3}} ^{\frac{2 \mu}{3}+\delta_{0}}$ is computable. Therefore, we subtract the low energy part from the full DiscG and plug back in the integration, which changes the lower limit to $\frac{2 \mu}{3}+\delta_{0}$. In our calculations, we will use $\delta_{0}=0$. See [6] for more discussions.
} 


\section{Geometric function theory for $O(N)$ model}

\subsection{Typically real functions}

A typically real function $f(z)$ satisfies $\operatorname{Im}[f(z)] \operatorname{Im}[z]>0, \quad \operatorname{Im}[z] \neq 0$, i.e. the imaginary part of the function is positive in the upper half plane, and negative in the lower half plane. We will consider the subclass of typically real functions, namely regular and typically real inside the unit disk $|z|<1$

$$
f(z)=z+\sum_{p=2}^{\infty} b_{p} z^{p}
$$

A schlicht function $f(z)$ (normalized $f(0)=0$ and $f^{\prime}(0)=1$, see [4] for a quick overview), which is univalent inside the unit disk $|z|<1$ with $b_{p} \in \mathbb{R}$, is also typically real function. The kernel $H$ is a function of this kind.

If $f(z)$ is a regular typically real function in $|z|<1$, then the coefficients should satisfy the bounds (see $[2,3]$ for proof)

$$
-\kappa_{p} \leq b_{p} \leq p .
$$

An important representation of typically real is the Robertson representation [35].

Robertson representation. In $|z|<1$ a regular function $F(z)$ is typically real if and only if it has the Robertson representation:

$$
F(z)=\int_{-1}^{1} d f(\eta) \frac{z}{1-2 \eta z+z^{2}}
$$

where the measure $f(\eta)$ is a non-decreasing function.

From the crossing symmetric dispersion relation, we get the full amplitude as an integral of discontinuity of the amplitude $\mathcal{A}\left(s_{1}, s^{(+)}\left(s_{1}, a\right)\right)$ times the kernel $H\left(s_{1}^{\prime} ; s_{2}, s_{3}\right)$. The kernel $H\left(s_{1}^{\prime} ; s_{2}, s_{3}\right)$ is a univalent typically real function. For some range of $a$, the $\mathcal{A}\left(s_{1}, s^{(+)}\left(s_{1}, a\right)\right)$ is non-negative. We will show below from these two facts that the full amplitude can be recast as Robertson representation; hence it is a typically real function.

\subsection{Typically real functions for $O(N)$ model}

We would consider the combinations

$$
\begin{aligned}
& \mathcal{F}_{0}(\tilde{z}, a)=\mathcal{G}_{0}\left(s_{1}, s_{2}\right)-\alpha_{0}^{(0)} \\
& \mathcal{F}_{1}(\tilde{z}, a)=\mathcal{G}_{1}\left(s_{1}, s_{2}\right)+\mathcal{G}_{0}\left(s_{1}, s_{2}\right)-\alpha_{0}^{(1)}-\alpha_{0}^{(0)} \\
& \mathcal{F}_{2}(\tilde{z}, a)=\mathcal{G}_{2}\left(s_{1}, s_{2}\right)+\frac{\mathcal{G}_{0}\left(s_{1}, s_{2}\right)}{3}-\alpha_{0}^{(2)}-\frac{\alpha_{0}^{(0)}}{3} .
\end{aligned}
$$

By definition then we can write $\left(\tilde{z}=z^{3}\right)$

$$
\mathcal{F}_{k}(\tilde{z}, a)=\sum_{n=1}^{\infty} \alpha_{n}^{(k)}(a) a^{2 n} \tilde{z}^{n} ; k=0,1,2
$$


with

$$
\begin{aligned}
\alpha_{p}^{(k)}(a) a^{2 p} & =\sum_{n=0}^{p} \sum_{m=0}^{n} \widehat{\mathcal{W}}_{n-m, m}^{(k)} a^{m}(-1)^{p-n}(-27)^{n} a^{2 n}\left(\begin{array}{c}
-2 n \\
p-n
\end{array}\right), \\
\widehat{\mathcal{W}}_{n-m, m}^{(k)} & =\left(1-\frac{2 \delta_{2, k}}{3}\right) \mathcal{W}_{n-m, m}^{(0)}+\delta_{1, k} \mathcal{W}_{n-m, m}^{(1)}+\delta_{2, k} \mathcal{W}_{n-m, m}^{(2)} .
\end{aligned}
$$

In [3] it was shown that $\mathcal{F}_{0}(\tilde{z}, a)$ is a typically real function from the positivity of the $\operatorname{Disc}_{s}\left[\mathcal{G}_{0}\left(s_{1}^{\prime} ; s_{2}^{(+)}\left(s_{1}^{\prime}, a\right)\right)\right]$.

The kernel can be written as in $\tilde{z}$ variables as

$$
H\left(s_{1}, s_{1}, s_{2}, s_{3}\right)=\frac{27 a^{2} \tilde{z}\left(2 s_{1}-3 a\right)}{27 a^{3} \tilde{z}-27 a^{2} \tilde{z} s_{1}-(\tilde{z}-1)^{2} s_{1}^{3}}=\sum_{n=0}^{\infty} \beta_{n}\left(a, s_{1}\right) \tilde{z}^{n} .
$$

We know from [4] that Kernel is a univalent function inside the unit disk. Notice that for $\beta_{1}\left(a, s_{1}\right)=\frac{27 a^{2}}{s_{1}^{3}}\left(3 a-2 s_{1}\right)<0$, we must have $a<4 \mu / 9$, since $s_{1} \geq 2 \mu / 3$ (see [3]). The dispersion relations for $\mathcal{F}_{k}(\tilde{z}, a)$ are given by

$$
\begin{aligned}
\mathcal{F}_{k}(\tilde{z}, a)= & \frac{1}{\pi} \int_{\frac{2 \mu}{3}}^{\infty} \frac{d s_{1}^{\prime}}{s_{1}^{\prime}} \operatorname{Disc}\left[\left(1-\frac{2 \delta_{2, k}}{3}\right) \mathcal{G}_{0}+\delta_{1, k} \mathcal{G}_{1}+\delta_{2, k} \mathcal{G}_{2}\right]\left(s_{1}^{\prime} ; s_{2}^{(+)}\left(s_{1}^{\prime}, a\right)\right) \\
& \times \frac{27 a^{2} \tilde{z}\left(2 s_{1}^{\prime}-3 a\right)}{27 a^{3} \tilde{z}-27 a^{2} \tilde{z} s_{1}^{\prime}-(\tilde{z}-1)^{2} s_{1}^{\prime 3}} .
\end{aligned}
$$

In the combination Disc $\left[\left(1-\frac{2 \delta_{2, k}}{3}\right) \mathcal{G}_{0}+\delta_{1, k} \mathcal{G}_{1}+\delta_{2, k} \mathcal{G}_{2}\right]$, if we use (2.10) and collect the coefficients of $\mathcal{A}^{(I)}\left(s_{1}, s^{(+)}\left(s_{1}, a\right)\right)$, we find that the coefficients are always positive for $s_{1}^{\prime} \geq$ $\frac{2 \mu}{3}, N \geq 3, \mu \geq 4$ for some range of $a$ : for $k=1,2$ the range of $a$ is $-\frac{s_{1}^{\prime}}{3}<a<\frac{s_{1}^{\prime}}{3}$, while for $k=0$ the range of $a$ is $-\frac{s_{1}^{\prime}}{3}<a<\frac{2 s_{1}^{\prime}}{3}$. Now since of the absorptive part of isospin amplitudes is always non-negative $\mathcal{A}^{(I)}\left(s_{1}^{\prime} ; s_{2}^{(+)}\left(s_{1}^{\prime}, a\right)\right)$ for $-\frac{2 \mu}{9}<a<\frac{2 \mu}{3}, s_{1}^{\prime} \geq \frac{2 \mu}{3}$. Therefore we find that the combination is always non-negative for ranges discussed above

$$
\operatorname{Disc}\left[\left(1-\frac{2 \delta_{2, k}}{3}\right) \mathcal{G}_{0}+\delta_{1, k} \mathcal{G}_{1}+\delta_{2, k} \mathcal{G}_{2}\right]\left(s_{1}^{\prime} ; s_{2}^{(+)}\left(s_{1}^{\prime}, a\right)\right) \geq 0 .
$$

Since $s_{1}^{\prime} \geq \frac{2 \mu}{3}$, therefore the range of $a$ in case of $k=0$ is given by $-\frac{2 \mu}{9}<a<\frac{4 \mu}{9}$, while in case of $k=1,2$ range of $a$ is given by $-\frac{2 \mu}{9}<a<\frac{2 \mu}{9}$. See appendix $\mathrm{C}$ for more clarifications.

We change the variable $s_{1}^{\prime}$ to $\eta$ by the equation

$$
-\frac{27 a^{3}}{\left(s_{1}^{\prime}\right)^{3}}+\frac{27 a^{2}}{\left(s_{1}^{\prime}\right)^{2}}-2=2 \eta .
$$

In this changed variable, we can write $\mathcal{F}_{k}(\tilde{z}, a)$ as (see [3] for more details)

$$
\frac{\mathcal{F}_{k}(\tilde{z}, a)}{\int_{-1}^{1} d \eta \overline{\operatorname{Disc}}_{k}(\eta, a)}=\int_{-1}^{1} d f_{k}(\eta) \frac{\tilde{z}}{1-2 \eta \tilde{z}+\tilde{z}^{2}}
$$

where

$$
d f_{k}(\eta)=\frac{\overline{\operatorname{Disc}}_{k}(\eta, a) d \eta}{\int_{-1}^{1} d \eta \overline{\operatorname{Disc}}_{k}(\eta, a)} .
$$


We have adopted the notation $\overline{\operatorname{Disc}}_{k}(\eta, a)$ for $\operatorname{Disc}\left[\left(1-\frac{2 \delta_{2, k}}{3}\right) \mathcal{G}_{0}+\delta_{1, k} \mathcal{G}_{1}+\delta_{2, k} \mathcal{G}_{2}\right]$ $\left(s_{1}^{\prime} ; s_{2}^{(+)}\left(s_{1}^{\prime}, a\right)\right)$, after changing the variable $s_{1}^{\prime} \rightarrow \eta$. Since the $\overline{\operatorname{Disc}}_{k}(\eta, a)$ are positive (for $s_{1}^{\prime} \geq \frac{2 \mu}{3}, N \geq 3, \mu \geq 4$ and range of $a$ in case of $k=0$ is given by $-\frac{2 \mu}{9}<a<\frac{4 \mu}{9}$, while in case of $k=1,2$ range of $a$ is given by $-\frac{2 \mu}{9}<a<\frac{2 \mu}{9}$ ) then

$$
f_{k}(\zeta)=\frac{\int_{-1}^{\zeta} \overline{\operatorname{Disc}}_{k}(\eta, a) d \eta}{\int_{-1}^{1} d \eta \overline{\operatorname{Disc}}_{k}(\eta, a)}
$$

are non-decreasing functions. We can conclude that functions $\mathcal{F}_{k}(\tilde{z}, a)$ are typically real functions.

\subsection{Rogosinski bounds on $\alpha_{p}^{(k)}(a)$}

Since functions $\mathcal{F}_{k}(\tilde{z}, a)$ are typically real functions, therefore we can readily write

$$
-\kappa_{n} \leq \frac{\alpha_{n}^{(k)}(a) a^{2 n}}{\alpha_{1}^{(k)}(a) a^{2}} \leq n, \text { for }\left\{\begin{array}{l}
k=0,-\frac{2 \mu}{9}<a<\frac{4 \mu}{9}, \\
k=1,2,-\frac{2 \mu}{9}<a<\frac{2 \mu}{9},
\end{array},\right.
$$

where

$$
\kappa_{p}=-\frac{\sin p \varphi_{p}}{\sin \varphi_{p}}, \quad \csc \left(\varphi_{p}\right)\left(p \cos \left(p \varphi_{p}\right)-\sin \left(p \varphi_{p}\right) \cot \left(\varphi_{p}\right)\right)=0 .
$$

For $p$ odd, $\pi / p<\varphi_{p}<3 \pi / 2 p$, while for $p$ even, $\varphi_{p}=\pi$ is only solution, giving $\kappa_{p}=p$. Notice the range of $a$ is different for different $k$ and for $k=1,2$, one will have to restrict to the cases $N \geq 3, \mu \geq 4$

\section{Geometric function theory constraints on pion amplitudes}

In this section, we discuss the geometric function theory constraints on the Pion amplitudes. We will consider the case $N=3, \mu=4$ in this section. For pion amplitudes, the functions $\mathcal{F}_{k}(\tilde{z}, a)$ are indeed typically real functions. It is more natural to work with $\widehat{\mathcal{W}}_{n-m, m}^{(k)}$ defined in (4.6).

\subsection{Bounds on coefficients $\widehat{\mathcal{W}}_{n-m, m}^{(k)}$}

Since the imaginary part of $\mathcal{F}_{k}(\tilde{z}, a)$ is positive for the range given in (4.14), therefore we note that

$$
\widehat{\mathcal{W}}_{n, 0}^{(k)} \geq 0 \text { for } \mu \geq 4 \text {. }
$$

This is true since here $m=0$ or we are considering the coefficients of $a^{0}$. Which enable us to compute $\widehat{\mathcal{W}}_{n, 0}^{(k)}$, which is given by

$$
\widehat{\mathcal{W}}_{n, 0}^{(k)}=\frac{1}{\pi} \int_{\frac{2 \mu}{3}}^{\infty} \frac{d s_{1}^{\prime}}{s_{1}^{\prime 2 n+1}}\left(2 \times \operatorname{Disc}\left[\left(1-\frac{2 \delta_{2, k}}{3}\right) \mathcal{G}_{0}+\delta_{1, k} \mathcal{G}_{1}+\delta_{2, k} \mathcal{G}_{2}\right]\left(s_{1}^{\prime} ; 0\right)\right) \geq 0 .
$$

Therefore we can write (derivation is similar to second equation of (3.1), see [6] for details)

$$
0 \leq \widehat{\mathcal{W}}_{n, 0}^{(k)} \leq \frac{\widehat{\mathcal{W}}_{n-1,0}^{(k)}}{\left(\delta_{0}+\frac{2 \mu}{3}\right)^{2}}
$$


All the bounds come in the form of $\frac{\widehat{\mathcal{W}}_{p, q}^{(k)}}{\widehat{\mathcal{W}}_{1,0}^{(k)}}$ (we can divide since $\left.\widehat{\mathcal{W}}_{1,0}^{(k)}>0\right)$. Therefore we will normalize $\widehat{\mathcal{W}}_{p, q}^{(k)}$ such that

$$
\widehat{\mathcal{W}}_{1,0}^{(k)}=1
$$

We can use the Rogosinski bounds on $\alpha_{p}^{(k)}(a) a^{2 p}$ and positivity bounds on the $\mathcal{W}_{p, q}^{(k)}$, to put two sided bounds on $\widehat{\mathcal{W}}_{p, q}^{(k)}$. Table 2 shows first few bounds on $\widehat{\mathcal{W}}_{p, q}^{(k)}$. For clarity, we write all the equations once again here.

\section{Rogosinski bounds on $\alpha_{p}^{(k)}(a) a^{2 p}$.}

$$
\begin{aligned}
-\kappa_{n} & \leq \frac{\alpha_{n}^{(k)}(a) a^{2 n}}{\alpha_{1}^{(k)}(a) a^{2}} \leq n, \text { for }\left\{\begin{array}{l}
k=0,-\frac{2 \mu}{9}<a<\frac{4 \mu}{9}, \\
k=1,2,-\frac{2 \mu}{9}<a<\frac{2 \mu}{9},
\end{array},\right. \\
\alpha_{p}^{(k)}(a) a^{2 p} & =\sum_{n=0}^{p} \sum_{m=0}^{n} \widehat{\mathcal{W}}_{n-m, m}^{(k)} a^{m}(-1)^{p-n}(-27)^{n} a^{2 n}\left(\begin{array}{c}
-2 n \\
p-n
\end{array}\right), \\
\widehat{\mathcal{W}}_{n-m, m}^{(k)} & =\left(1-\frac{2 \delta_{2, k}}{3}\right) \mathcal{W}_{n-m, m}^{(0)}+\delta_{1, k} \mathcal{W}_{n-m, m}^{(1)}+\delta_{2, k} \mathcal{W}_{n-m, m}^{(2)} .
\end{aligned}
$$

\section{Positivity conditions.}

$$
\begin{aligned}
& \sum_{r=0}^{m} \chi_{n, r, m}^{(0)}\left(\mu, \delta_{0}\right) \mathcal{W}_{n-r, r}^{(0)} \geq 0, \quad \sum_{r=0}^{m}\left(\Upsilon_{n, r, m}^{(1)}\left(\mu, \delta_{0}\right) \mathcal{W}_{n-r, r}^{(1)}+\chi_{n, r, m}^{(1)}\left(\mu, \delta_{0}\right) \mathcal{W}_{n-r, r}^{(0)}\right) \geq 0 \\
& \sum_{r=0}^{m}\left(\Upsilon_{n, r, m}^{(2)}\left(\mu, \delta_{0}\right) \mathcal{W}_{n-r, r}^{(2)}+\chi_{n, r, m}^{(2)}\left(\mu, \delta_{0}\right) \mathcal{W}_{n-r, r}^{(0)}\right) \geq 0, \quad 0 \leq \widehat{\mathcal{W}}_{n, 0}^{(k)} \leq \frac{\widehat{\mathcal{W}}_{n-1,0}^{(k)}}{\left(\delta_{0}+\frac{2 \mu}{3}\right)^{2}}
\end{aligned}
$$

In the above equations, we will consider $m=1,2,3, \ldots n$. The Taylor coefficients of the amplitude around the crossing symmetric point (appearing on $\alpha_{n}^{(k)}(a) a^{2 n}$ ) can be bounded by using up to $n$-th equations in Rogosinski bounds on $\alpha_{n}^{(k)}(a) a^{2 n}$; in that case, one must use up to $n$-th positivity conditions with $m=1,2,3, \ldots n$.

The $\boldsymbol{n}=\mathbf{2}$ bounds. The simplest exercise is for the $n=2$ bounds. We put $n=2$ in equation (5.5) and $n=2, m=1,2$ in equation (5.6), then maximize and minimize ${ }^{9}$ with respect to the coefficients $\widehat{\mathcal{W}}_{n-m, m}^{(k)}$ that appear in those equations. Table 1 contains the bounds we have found.

Using the $n=2$ constraints, we find the finite region of theory space as depicted in figure 1. We have also indicated some of the known theories.

The $\boldsymbol{n}=\mathbf{3}$ bounds. We put $n=2,3$ in equation (5.5) and $n=2,3, m=1,2,3$ in equation (5.6), then Maximize and Minimize with respect to the coefficients $\widehat{\mathcal{W}}_{n-m, m}^{(k)}$ those appear in these equations, we find the bounds given in 2.

Once we have bounds on $\widehat{\mathcal{W}}_{p, q}^{(k)}$, in principle we can bound Taylor coefficients of the amplitude around the crossing symmetric point of any physical reactions. Below we show such an example.

\footnotetext{
${ }^{9}$ In Mathematica, we define ImplicitRegion, and we find the bounds on the Taylor coefficients using RegionBounds.
} 


\begin{tabular}{|l|l|l|l|l|l|l|l|l|}
\hline$\widehat{\mathcal{W}}_{p, q}^{(0)}$ & Lower & Upper & $\widehat{\mathcal{W}}_{p, q}^{(1)}$ & Lower & Upper & $\widehat{\mathcal{W}}_{p, q}^{(2)}$ & Lower & Upper \\
\hline$\widehat{\mathcal{W}}_{1,1}^{(0)}$ & -0.131836 & 0.105469 & $\widehat{\mathcal{W}}_{1,1}^{(1)}$ & -0.211282 & 0.217698 & $\widehat{\mathcal{W}}_{1,1}^{(2)}$ & -0.147396 & 0.217698 \\
\hline$\widehat{\mathcal{W}}_{2,0}^{(0)}$ & 0 & 0.140625 & $\widehat{\mathcal{W}}_{2,0}^{(1)}$ & 0 & 0.140625 & $\widehat{\mathcal{W}}_{2,0}^{(2)}$ & -0.0000601055 & 0.140625 \\
\hline$\widehat{\mathcal{W}}_{0,2}^{(0)}$ & -0.0889893 & 0.0395508 & $\widehat{\mathcal{W}}_{0,2}^{(1)}$ & -0.180231 & 0.243389 & $\widehat{\mathcal{W}}_{0,2}^{(2)}$ & -0.165266 & 0.243427 \\
\hline$\widehat{\mathcal{W}}_{0,1}^{(0)}$ & -0.562500 & 1.12500 & $\widehat{\mathcal{W}}_{0,1}^{(1)}$ & -1.13924 & 1.12500 & $\widehat{\mathcal{W}}_{0,1}^{(2)}$ & -1.09570 & 1.12500 \\
\hline
\end{tabular}

Table 1. Bounds on $\widehat{\mathcal{W}}_{p, q}^{(k)}$ for $n=2$ in equation (4.14). We use the normalizations $\widehat{\mathcal{W}}_{1,0}^{(k)}=1$,. The normalization can be restored via replacing $\widehat{\mathcal{W}}_{p, q}^{(k)} \rightarrow \frac{\widehat{\mathcal{W}}_{p, q}^{(k)}}{\widehat{\mathcal{W}}_{1,0}^{(k)}}$.
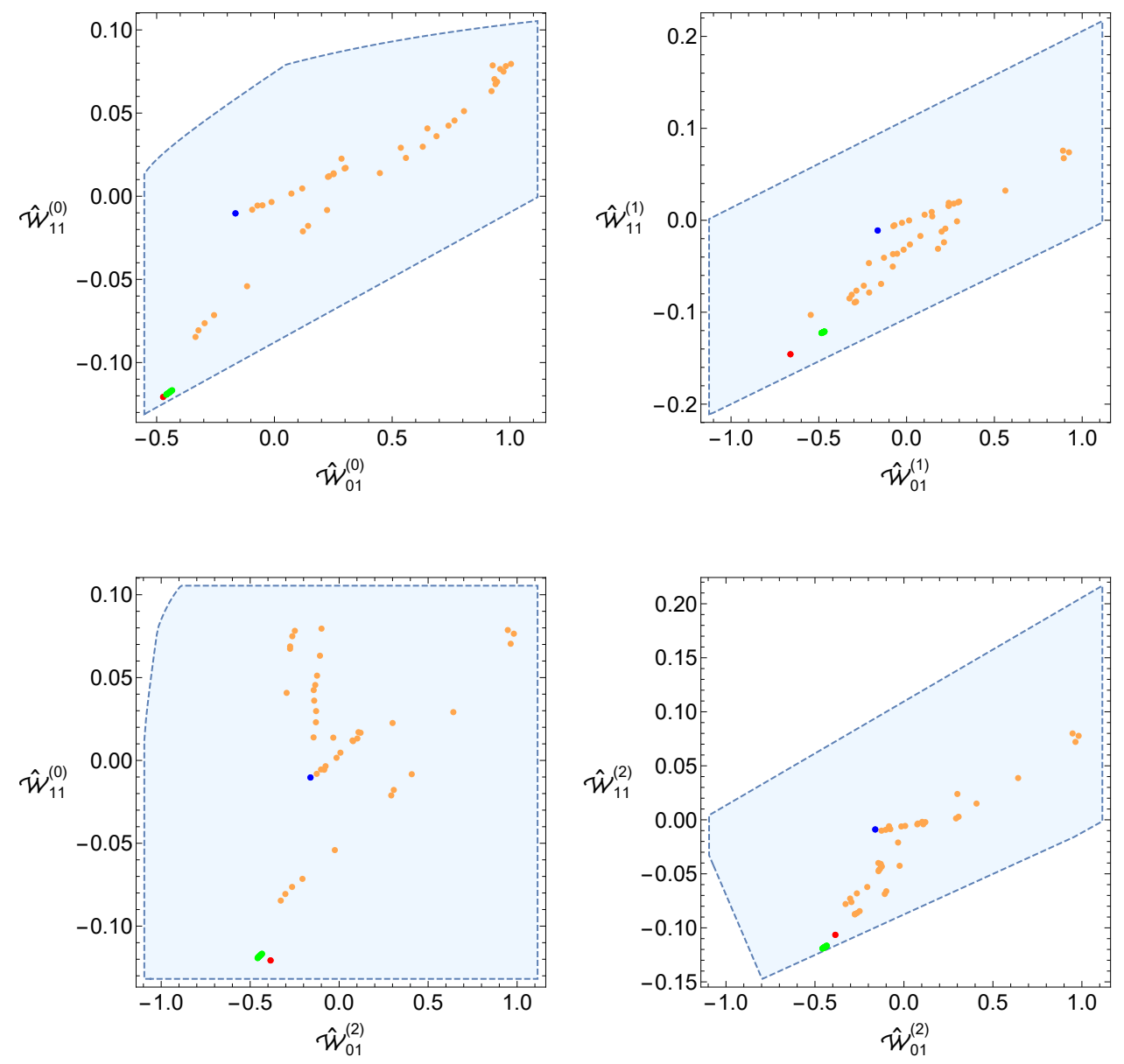

Figure 1. The light blue region is the theory space allowed by positivity and typically realness. Some of the known theories has been indicated. Green region is the $O(3)$ Lovelace-Shapiro model (A.1) with $\beta^{\prime}=\frac{1}{m_{\rho}^{2}}\left(1-\beta_{0}\right)$ and $\beta_{0}$ varies from $0.465<\beta_{0}<0.489$ (see [36]). The red dot is the $O(3)$ Lovelace-Shapiro model (A.1) with $\beta_{0}=\beta^{\prime}=1 / 2$. The blue dot is the 2-loop chiral perturbation theory with parameters are taken from experimental values. The orange regions are obtained from the S-matrix bootstrap amplitude for the upper river boundaries [36, 37]. 


\begin{tabular}{|l|l|l|l|l|l|l|l|l|}
\hline$\widehat{\mathcal{W}}_{p, q}^{(0)}$ & Lower & Upper & $\widehat{\mathcal{W}}_{p, q}^{(1)}$ & Lower & Upper & $\widehat{\mathcal{W}}_{p, q}^{(2)}$ & Lower & Upper \\
\hline$\widehat{\mathcal{W}}_{1,1}^{(0)}$ & -0.131836 & 0.105469 & $\widehat{\mathcal{W}}_{1,1}^{(1)}$ & -0.211282 & 0.217698 & $\widehat{\mathcal{W}}_{1,1}^{(2)}$ & -0.147396 & 0.217698 \\
\hline$\widehat{\mathcal{W}}_{2,0}^{(0)}$ & 0 & 0.140625 & $\widehat{\mathcal{W}}_{2,0}^{(1)}$ & 0 & 0.140625 & $\widehat{\mathcal{W}}_{2,0}^{(2)}$ & 0 & \\
\hline$\widehat{\mathcal{W}}_{0,2}^{(0)}$ & -0.0889893 & 0.0395508 & $\widehat{\mathcal{W}}_{0,2}^{(1)}$ & -0.180231 & 0.243389 & $\widehat{\mathcal{W}}_{0,2}^{(2)}$ & -0.165266 & 0.243427 \\
\hline$\widehat{\mathcal{W}}_{0,1}^{(0)}$ & -0.562500 & 1.12500 & $\widehat{\mathcal{W}}_{0,1}^{(1)}$ & -1.13924 & 1.12500 & $\widehat{\mathcal{W}}_{0,1}^{(2)}$ & -1.09570 & 1.12500 \\
\hline$\widehat{\mathcal{W}}_{1,2}^{(0)}$ & -0.0194715 & 0.0114256 & $\widehat{\mathcal{W}}_{1,2}^{(1)}$ & -0.0372450 & 0.0475034 & $\widehat{\mathcal{W}}_{1,2}^{(2)}$ & -0.0291138 & 0.0648537 \\
\hline$\widehat{\mathcal{W}}_{2,1}^{(0)}$ & -0.0259552 & 0.0104932 & $\widehat{\mathcal{W}}_{2,1}^{(1)}$ & -0.0371815 & 0.100608 & $\widehat{\mathcal{W}}_{2,1}^{(2)}$ & -0.0290552 & 0.100608 \\
\hline$\widehat{\mathcal{W}}_{3,0}^{(0)}$ & 0 & 0.0197754 & $\widehat{\mathcal{W}}_{3,0}^{(1)}$ & 0 & 0.0197754 & $\widehat{\mathcal{W}}_{3,0}^{(2)}$ & -0.0423541 & 0.0197754 \\
\hline$\widehat{\mathcal{W}}_{0,3}^{(0)}$ & -0.00587333 & 0.00708816 & $\widehat{\mathcal{W}}_{0,3}^{(1)}$ & -0.110787 & 0.0599811 & $\widehat{\mathcal{W}}_{0,3}^{(2)}$ & -0.110787 & 0.0546385 \\
\hline
\end{tabular}

Table 2. Bounds on $\widehat{\mathcal{W}}_{p, q}^{(k)}$ for $n=3$.

Even though we work with $N=3$, our bounds (table 1,2) do not depend on $N$. The $N$ dependant factor only enters through the positivity condition (3.3), which goes away when considering the ratio.

\subsection{Bounds on Taylor coefficients of physical amplitudes}

The $A\left(s_{1} \mid s_{2}, s_{3}\right)$ is symmetric under exchange of $s_{2} \leftrightarrow s_{3}$. Therefore without lose of generality, we can write

$$
A\left(s_{1} \mid s_{2}, s_{3}\right)=\sum_{p=0, q=0}^{\infty} \mathcal{C}_{p, q}\left(-s_{2} s_{3}\right)^{p}\left(-s_{1}\right)^{q}
$$

Once we have the bounds on $\widehat{\mathcal{W}}_{p, q}^{(k)}$, using the isospin amplitudes, namely using (2.13), we can put bounds on Taylor coefficients of any physical amplitudes around the crossing symmetric point. For example, for the reaction $\pi^{+}+\pi^{-} \rightarrow \pi^{0}+\pi^{0}$ the amplitude is

$$
\begin{aligned}
\mathcal{M}^{\left(\pi^{+}+\pi^{-} \rightarrow \pi^{0}+\pi^{0}\right)}\left(s_{1}, s_{2}\right) & =\frac{1}{3}\left(\mathcal{M}^{0}\left(s_{1}, s_{2}\right)-\mathcal{M}^{2}\left(s_{1}, s_{2}\right)\right)=A\left(s_{1} \mid s_{2}, s_{3}\right) \\
& =\sum_{p=0, q=0}^{\infty} \mathcal{C}_{p, q}\left(-s_{2} s_{3}\right)^{p}\left(-s_{1}\right)^{q}
\end{aligned}
$$

Using the equation (2.13) and the expansion (2.14), we find that

$$
\begin{aligned}
\frac{1}{3}\left(\mathcal{M}^{0}\left(s_{1}, s_{2}\right)-\mathcal{M}^{2}\left(s_{1}, s_{2}\right)\right)=A\left(s_{1} \mid s_{2}, s_{3}\right)= & \frac{1}{9}\left(3 \mathcal{G}_{0}\left(s_{1}, s_{2}\right)+3 s_{1} \mathcal{G}_{1}\left(s_{1}, s_{2}\right)\right. \\
& \left.+\left(-s_{1}^{2}+2 s_{2} s_{1}+2 s_{2}^{2}\right) \mathcal{G}_{2}\left(s_{1}, s_{2}\right)\right)
\end{aligned}
$$




\begin{tabular}{|l|l|l|l|l|l|l|l|l|}
\hline & Lower & Upper & & Lower & Upper & & Lower & Upper \\
\hline $\mathcal{C}_{0,4}$ & -0.0740741 & -0.0271991 & $\mathcal{C}_{1,2}$ & -0.680673 & 0.730324 & $\mathcal{C}_{2,1}$ & -0.373698 & 0.417643 \\
\hline $\mathcal{C}_{0,5}$ & -0.046875 & 0.046875 & $\mathcal{C}_{1,3}$ & -0.292318 & 0.283529 & $\mathcal{C}_{2,2}$ & -0.150892 & 0.196345 \\
\hline $\mathcal{C}_{0,6}$ & -0.015625 & 0.0118068 & $\mathcal{C}_{1,4}$ & -0.105584 & 0.136287 & $\mathcal{C}_{2,3}$ & -0.166635 & 0.130623 \\
\hline $\mathcal{C}_{0,7}$ & -0.0065918 & 0.0065918 & $\mathcal{C}_{1,5}$ & -0.0435567 & 0.0574986 & $\mathcal{C}_{3,0}$ & -0.01043 & 0.0378418 \\
\hline $\mathcal{C}_{1,1}$ & -0.375 & 0.1875 & $\mathcal{C}_{2,0}$ & 0.148148 & 0.195023 & $\mathcal{C}_{3,1}$ & -0.0682325 & 0.0558107 \\
\hline
\end{tabular}

Table 3. Bounds on $\mathcal{C}_{p, q}$. Note that $\widehat{\mathcal{W}}_{1,0}^{(k)}=1$ in our normalization. Also note that $\mathcal{C}_{p, q}$ which contains the subtraction constants $\widehat{\mathcal{W}}_{0,0}^{(k)}$ can't be bounded. In order to restore the normalization and get the two sided bounds in terms of $\widehat{\mathcal{W}}_{1,0}^{(k)}$ one would have to replace $\widehat{\mathcal{W}}_{p, q}^{(k)} \rightarrow \frac{\widehat{\mathcal{W}}_{p, q}^{(k)}}{\widehat{\mathcal{W}}_{1,0}^{(k)}}$ in table 2 and use in equation (5.10).

Therefore we can write $\mathcal{C}_{p, q}$ as a combinations of $\widehat{\mathcal{W}}_{p, q}^{(k)}$. For example

$$
\begin{aligned}
& \mathcal{C}_{0,0}=\frac{1}{3} \widehat{\mathcal{W}}_{0,0}^{(0)}, \mathcal{C}_{0,1}=\frac{1}{3}\left(\widehat{\mathcal{W}}_{0,0}^{(0)}-\widehat{\mathcal{W}}_{0,0}^{(1)}\right), \mathcal{C}_{0,2}=\frac{1}{27}\left(\widehat{\mathcal{W}}_{0,0}^{(0)}+9 \widehat{\mathcal{W}}_{1,0}^{(0)}-3 \widehat{\mathcal{W}}_{0,0}^{(2)}\right), \mathcal{C}_{0,3}=\frac{1}{3}\left(\widehat{\mathcal{W}}_{1,0}^{(0)}-\widehat{\mathcal{W}}_{1,0}^{(1)}\right) \\
& \mathcal{C}_{0,4}=\frac{1}{27}\left(\widehat{\mathcal{W}}_{1,0}^{(0)}+9 \widehat{\mathcal{W}}_{2,0}^{(0)}-3 \widehat{\mathcal{W}}_{1,0}^{(2)}\right), \mathcal{C}_{0,5}=\frac{1}{3}\left(\widehat{\mathcal{W}}_{2,0}^{(0)}-\widehat{\mathcal{W}}_{2,0}^{(1)}\right), \mathcal{C}_{0,6}=\frac{1}{27}\left(\widehat{\mathcal{W}}_{2,0}^{(0)}+9 \widehat{\mathcal{W}}_{3,0}^{(0)}-3 \widehat{\mathcal{W}}_{2,0}^{(2)}\right) \\
& \mathcal{C}_{0,7}=\frac{1}{3}\left(\widehat{\mathcal{W}}_{3,0}^{(0)}-\widehat{\mathcal{W}}_{3,0}^{(1)}\right), \mathcal{C}_{1,0}=\frac{1}{27}\left(-2 \widehat{\mathcal{W}}_{0,0}^{(0)}+9 \widehat{\mathcal{W}}_{1,0}^{(0)}+6 \widehat{\mathcal{W}}_{0,0}^{(2)}\right), \mathcal{C}_{1,1}=\frac{1}{3}\left(-\widehat{\mathcal{W}}_{0,1}^{(0)}+\widehat{\mathcal{W}}_{1,0}^{(0)}-\widehat{\mathcal{W}}_{1,0}^{(1)}\right) \\
& \mathcal{C}_{1,2}=\frac{1}{9}\left(-3 \widehat{\mathcal{W}}_{0,1}^{(0)}-\frac{1}{3} \widehat{\mathcal{W}}_{1,0}^{(0)}+6 \widehat{\mathcal{W}}_{2,0}^{(0)}+3 \widehat{\mathcal{W}}_{0,1}^{(1)}+\widehat{\mathcal{W}}_{1,0}^{(2)}\right), \mathcal{C}_{1,3}=\frac{1}{9}\left(-\frac{1}{3} \widehat{\mathcal{W}}_{0,1}^{(0)}-3 \widehat{\mathcal{W}}_{1,1}^{(0)}+6 \widehat{\mathcal{W}}_{2,0}^{(0)}-6 \widehat{\mathcal{W}}_{2,0}^{(1)}+\widehat{\mathcal{W}}_{0,1}^{(2)}\right) \\
& \mathcal{C}_{1,4}=\frac{1}{3}\left(\widehat{\mathcal{W}}_{1,1}^{(1)}-\widehat{\mathcal{W}}_{1,1}^{(0)}\right)+\widehat{\mathcal{W}}_{3,0}^{(0)}, \mathcal{C}_{1,5}=-\frac{1}{27} \widehat{\mathcal{W}}_{1,1}^{(0)}-\frac{1}{3} \widehat{\mathcal{W}}_{2,1}^{(0)}+\widehat{\mathcal{W}}_{3,0}^{(0)}-\widehat{\mathcal{W}}_{3,0}^{(1)}+\frac{1}{9} \widehat{\mathcal{W}}_{1,1}^{(2)} \\
& \mathcal{C}_{2,0}=\frac{1}{27}\left(-2 \widehat{\mathcal{W}}_{1,0}^{(0)}+9 \widehat{\mathcal{W}}_{2,0}^{(0)}+6 \widehat{\mathcal{W}}_{1,0}^{(2)}\right) \mathcal{C}_{2,1}=\frac{1}{27}\left(-9\left(\widehat{\mathcal{W}}_{1,1}^{(0)}-\widehat{\mathcal{W}}_{2,0}^{(0)}+\widehat{\mathcal{W}}_{2,0}^{(1)}\right)+2 \widehat{\mathcal{W}}_{0,1}^{(0)}-6 \widehat{\mathcal{W}}_{0,1}^{(2)}\right) \\
& \mathcal{C}_{2,2}=\frac{1}{3}\left(\widehat{\mathcal{W}}_{0,2}^{(0)}-\widehat{\mathcal{W}}_{1,1}^{(0)}-\frac{1}{3} \widehat{\mathcal{W}}_{2,0}^{(0)}+3 \widehat{\mathcal{W}}_{3,0}^{(0)}+\widehat{\mathcal{W}}_{1,1}^{(1)}+\widehat{\mathcal{W}}_{2,0}^{(2)}\right) \\
& \mathcal{C}_{2,3}=\frac{1}{27}\left(-9\left(2 \widehat{\mathcal{W}}_{2,1}^{(0)}-3 \widehat{\mathcal{W}}_{3,0}^{(0)}+3 \widehat{\mathcal{W}}_{3,0}^{(1)}\right)+9 \widehat{\mathcal{W}}_{0,2}^{(0)}+\widehat{\mathcal{W}}_{1,1}^{(0)}-9 \widehat{\mathcal{W}}_{0,2}^{(1)}-3 \widehat{\mathcal{W}}_{1,1}^{(2)}\right) \\
& \mathcal{C}_{3,0}=\frac{1}{27}\left(-2 \widehat{\mathcal{W}}_{2,0}^{(0)}+9 \widehat{\mathcal{W}}_{3,0}^{(0)}+6 \widehat{\mathcal{W}}_{2,0}^{(2)}\right), \mathcal{C}_{3,1}=\frac{1}{27}\left(-9\left(\widehat{\mathcal{W}}_{2,1}^{(0)}-\widehat{\mathcal{W}}_{3,0}^{(0)}+\widehat{\mathcal{W}}_{3,0}^{(1)}\right)+2 \widehat{\mathcal{W}}_{1,1}^{(0)}-6 \widehat{\mathcal{W}}_{1,1}^{(2)}\right) . \quad(5.10)
\end{aligned}
$$

Using the bounds on $\widehat{\mathcal{W}}_{p, q}^{(k)}$, we can get bounds on $\mathcal{C}_{p, q}$. For example first few are shown in the table 3 We have used the normalization $\widehat{\mathcal{W}}_{1,0}^{(k)}=1$. We restore the normalization and get the two sided bounds in terms of $\widehat{\mathcal{W}}_{1,0}^{(k)}$, we replace $\widehat{\mathcal{W}}_{p, q}^{(k)} \rightarrow \frac{\widehat{\mathcal{W}}_{p, q}^{(k)}}{\widehat{\mathcal{W}}_{1,0}^{(k)}}$ in table 2 and use that in equation (5.10). For example

$$
\begin{aligned}
\frac{1}{3}\left(-\frac{1}{8} \widehat{\mathcal{W}}_{1,0}^{(0)}-\widehat{\mathcal{W}}_{1,0}^{(1)}\right) & <\mathcal{C}_{1,1}<\frac{1}{3}\left(\frac{25}{16} \widehat{\mathcal{W}}_{1,0}^{(0)}-\widehat{\mathcal{W}}_{1,0}^{(1)}\right), \\
\frac{1}{9}\left(\frac{1}{24}(-89) \widehat{\mathcal{W}}_{1,0}^{(0)}-\frac{270}{79} \widehat{\mathcal{W}}_{1,0}^{(1)}+\widehat{\mathcal{W}}_{1,0}^{(2)}\right) & <\mathcal{C}_{1,2}<\frac{1}{9}\left(\frac{211}{96} \widehat{\mathcal{W}}_{1,0}^{(0)}+\frac{27}{8} \widehat{\mathcal{W}}_{1,0}^{(1)}+\widehat{\mathcal{W}}_{1,0}^{(2)}\right) .
\end{aligned}
$$

If we put $\widehat{\mathcal{W}}_{1,0}^{(k)}=1$, then we recover the results that are given in 3 . Using the constraints in the table 3, we find the finite region of theory space as depicted in figure 2. Some of the known theories are also indicated.

For the 2-loop chiral perturbation theory and the $O(3)$ Lovelace-Shapiro model, we have calculated some of the $\widehat{\mathcal{W}}_{p, q}^{(k)}$ and $\mathcal{C}_{p, q}$ with our normalization $\widehat{\mathcal{W}}_{1,0}^{(k)}=1$, which are presented in the appendix D. 

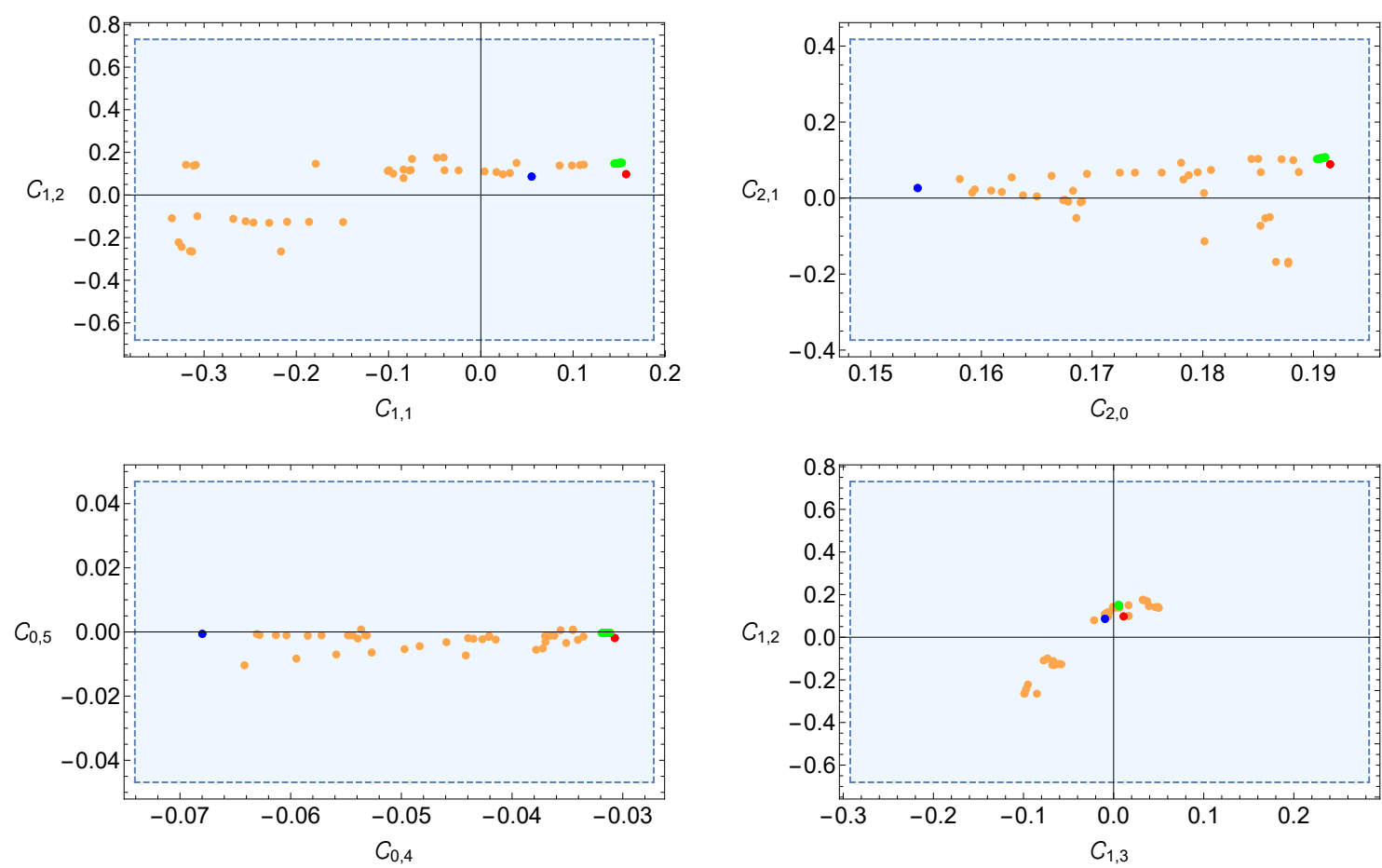

Figure 2. The light blue region is the theory space allowed by the constraints in the table 3 . Some of the known theories are indicated. Green region is the $O(3)$ Lovelace-Shapiro model (A.1) with $\beta^{\prime}=\frac{1}{m_{\rho}^{2}}\left(1-\beta_{0}\right)$ and $\beta_{0}$ varies from $0.465<\beta_{0}<0.489$ (see [36]). The red dot is the $O(3)$ Lovelace-Shapiro model (A.1) with $\beta_{0}=\beta^{\prime}=1 / 2$. The blue dot is the 2-loop chiral perturbation theory with parameters are taken from experimental values. The orange regions are obtained from the S-matrix bootstrap amplitude for the upper river boundaries [36, 37].

\section{$6 \quad$ Summary and future directions}

In this paper, we have applied the techniques in geometric function theory, namely typically real functions to 2-2 scattering amplitudes with global $O(N)$ symmetry. We have used the fully crossing symmetric dispersion relation as in $[5,6]$.

The main results of the paper are the following:

- In the case of theory with $O(N)$ global symmetry, there exist three independent sets of fully crossing symmetric combinations ( see (2.4), (2.5), (2.6)) of isospin amplitudes, which was studied in [32]. We have written down three collections of fully crossing symmetric dispersion relations in $z$-variable for fixed $a$.

- The dispersion relations empowered us to derive three sets of independent sum rules. These new sum rules arise because of the cancellation of unphysical powers of $x$.

- We have written down the partial wave expansion for the dispersion relations through partial wave expansion of the isospin amplitudes (see (2.17)). These partial wave expansions allow us to derive of three novel sets of positivity conditions on the Taylor 
coefficients of amplitudes around the crossing symmetric point (see (3.1), (3.3), (3.4)) employing the unitarity and positivity of Gegenbauer polynomials.

- We find three kinds of typically real functions for the $O(N)$ model (4.4). Practising the dispersion relations and positivity of the absorptive part of the isospin amplitude for a fixed range of $a$, we have shown that these three functions (4.4) exhibit the Robertson representation (4.13). Robertson representation is a necessary and sufficient condition for typically real functions. Hence we have concluded that these three functions (4.4) are typically real.

- Typically real-ness of the functions (4.4) enable us to formulate the BieberbachRogosinski inequalities for typically real functions (4.14).

- The Bieberbach-Rogosinski inequalities and the positivity conditions permit us to write down two-sided bounds on the three groups of Taylor coefficients for pion scattering (table 2). These three assortments of bounds allow us to establish bounds on Taylor coefficients of any physical amplitudes around the crossing symmetric point (see for example table 3). Our bounds presented in table 2, do not depend on $N$.

- We have supported our bounds by comparing them against know theories, namely 2-loop chiral perturbative amplitude with experimental input on the $b_{i}$ parameters and the $O(3)$ Lovelace-Shapiro model.

Here are our immediate future directions:

- These three sets of dispersion relations can be employed in the matter of CFT Mellin amplitude to make connections with Polyakov Mellin Bootstrap [38-44]. The upcoming work of [45] will discuss the Witten block expansion. The upcoming work of [46] will show that a crossing antisymmetric correlator could be expanded in a manifestly crossing antisymmetric basis by introducing a crossing antisymmetric dispersion relation.

- An exciting application will be to see the implications of our sum rules (2.21) to the CFT correlators of charged fields discussed in [47] and the functionals therein.

- Another charming area worth exploring is CFT Mellin amplitudes in light of geometric function theory. It will be interesting to relate CFT typically real-ness with swampland conditions considered recently in [48, 49] as well as the tricky correlator bounds found in [50]. It will be very interesting to relate the geometric function theory analysis to the positive geometry of CFT, discussed in [51-53].

- It will be appealing to see the implications of our bounds and sum rules (2.21) to S-matrix bootstrap for pion amplitudes [36, 37, 54] and to the dual the S-matrix bootstrap [55-58]. 


\section{Acknowledgments}

We thank Aninda Sinha for suggesting the problem and numerous helpful discussions. We thank Kausik Ghosh, Parthiv Haldar, Apratim Kaviraj, Prashanth Raman for their comments on the draft. We also thank Shaswat Tiwari for helping us with the S-matrix bootstrap data.

\section{A Verification of various formulas against $O(3)$ Lovelace-Shapiro model}

In this section, we will verify our various formulas for the Lovelace-Shapiro model [36, 59$61]$.

$$
A\left(s_{1} \mid s_{2}, s_{3}\right)=\frac{\Gamma(1-\beta(s)) \Gamma(1-\beta(t))}{\Gamma(1-\beta(s)-\beta(t))} .
$$

with $\beta(s)=\beta_{0}+\beta^{\prime} s, \quad \beta\left(m_{\rho}^{2}\right)=1$.

For demonstration purpose, we will choose $\beta_{0}=\beta^{\prime}=\frac{1}{2}$. Lovelace-Shapiro model, in our convention, we write

$$
A^{(L S)}\left(s_{1}, s_{2}\right)=A^{(L S)}\left(s_{1} \mid s_{2}, s_{3}\right)=\frac{\Gamma\left(\frac{1}{2}-\frac{s_{1}}{2}\right) \Gamma\left(\frac{1}{2}-\frac{s_{2}}{2}\right)}{\Gamma\left(-\frac{s_{1}}{2}-\frac{s_{2}}{2}\right)},
$$

and

$$
\begin{aligned}
& \mathcal{M}_{(L S)}^{(0)}\left(s_{1}, s_{2}\right)=\frac{3}{2}\left(A^{(L S)}\left(s_{1}, s_{2}\right)+A^{(L S)}\left(s_{1}, s_{3}\right)\right)-\frac{1}{2} A^{(L S)}\left(s_{2}, s_{3}\right), \\
& \mathcal{M}_{(L S)}^{(1)}\left(s_{1}, s_{2}\right)=A^{(L S)}\left(s_{1}, s_{2}\right)-A^{(L S)}\left(s_{1}, s_{3}\right), \quad \mathcal{M}_{(L S)}^{(2)}\left(s_{1}, s_{2}\right)=A^{(L S)}\left(s_{2}, s_{3}\right) .
\end{aligned}
$$

\section{A.1 Dispersion relation}

In this section, we verify the dispersion integral (2.8) against Lovelace-Shapiro model. We plug (A.3) in (2.4), (2.5), (2.6), to get $\mathcal{G}_{k}\left(s_{1}, s_{2}\right)$. We calculate the discontinuity $\operatorname{Disc} \mathcal{G}_{k}\left(s_{1}, s_{2}\right)$. Then we plug back that calculated $\operatorname{Disc} \mathcal{G}_{k}\left(s_{1}, s_{2}\right)$ in formula $(2.8)$ and check if the l.h.s. is equal to r.h.s. i.e. if the dispersion relations are correct. The subtraction constants $\alpha_{0}^{(k)}$ are given by

$$
\alpha_{0}^{(0)}=0, \alpha_{0}^{(1)}=-\frac{3 \pi}{2}, \alpha_{0}^{(2)}=\frac{-3 \pi \log (4)}{4} .
$$

The $s$-channel discontinuities are given by

$$
\begin{aligned}
& \operatorname{Disc} \mathcal{G}_{0}\left(s_{1}, s_{2}^{(+)}\left(s_{1}, a\right)\right)=\sum_{k=0}^{\infty} \pi \delta\left(s_{1}-2 k-1\right) \frac{(-1)^{k}\left(-\frac{\Gamma\left(\frac{1}{4}(-2 k(\lambda-1)-\lambda+3)\right)}{\Gamma\left(-\frac{1}{4}(2 k+1)(\lambda+1)\right)}-\frac{\Gamma\left(\frac{1}{4}(\lambda+2 k(\lambda+1)+3)\right)}{\Gamma\left(\frac{1}{4}(2 k+1)(\lambda-1)\right)}\right)}{k !}, \\
& \operatorname{Disc} \mathcal{G}_{1}\left(s_{1}, s_{2}^{(+)}\left(s_{1}, a\right)\right)=\sum_{k=0}^{\infty} \pi \delta\left(s_{1}-2 k-1\right) \frac{3(-1)^{k} \lambda(a-2 k-1)}{(2 k+1)(3 a+2 k+1)(-3 a+4 k+2) \Gamma(k+1)} \\
& \times\left(\frac{((2 k+1)(\lambda+1)-a(\lambda+3)) \Gamma\left(\frac{1}{4}(-2 k(\lambda-1)-\lambda+3)\right)}{\Gamma\left(-\frac{1}{4}(2 k+1)(\lambda+1)\right)}+\frac{((2 k+1)(\lambda-1)-a(\lambda-3)) \Gamma\left(\frac{1}{4}(\lambda+2 k(\lambda+1)+3)\right)}{\Gamma\left(\frac{1}{4}(2 k+1)(\lambda-1)\right)}\right), \\
& \operatorname{Disc}_{2}\left(s_{1}, s_{2}^{(+)}\left(s_{1}, a\right)\right)=\sum_{k=0}^{\infty} \pi \delta\left(s_{1}-2 k-1\right) \frac{12(-1)^{k}\left(-\frac{\Gamma\left(\frac{1}{4}(-2 k(\lambda-1)-\lambda+3)\right)}{(\lambda+3) \Gamma\left(-\frac{1}{4}(2 k+1)(\lambda+1)\right)}-\frac{\Gamma\left(\frac{1}{4}(\lambda+2 k(\lambda+1)+3)\right)}{(\lambda-3) \Gamma\left(\frac{1}{4}(2 k+1)(\lambda-1)\right)}\right)}{(2 k+1)^{2} k ! \sqrt{\frac{4 a}{-a+2 k+1}+1}},
\end{aligned}
$$




\begin{tabular}{|l|l|l|l|}
\hline $\mathcal{G}_{k}\left(s_{1}, s_{2}\right)$ & Exact & $k_{\max }=100$ & $k_{\max }=150$ \\
\hline $\mathcal{G}_{0}\left(s_{1}=\frac{1}{22}, s_{2}=\frac{i}{13}\right)$ & $0.0038224+0.0035656 \mathrm{i}$ & $0.00382198+0.0035653 \mathrm{i}$ & $0.0038222+0.0035654 \mathrm{i}$ \\
\hline $\mathcal{G}_{0}\left(s_{1}=\frac{1}{2}-i, s_{2}=1+\frac{i}{3}\right)$ & $0.939177-0.790044 \mathrm{i}$ & $0.935403-0.796079 \mathrm{i}$ & $0.936207-0.794022 \mathrm{i}$ \\
\hline $\mathcal{G}_{1}\left(s_{1}=\frac{1}{22}, s_{2}=\frac{i}{13}\right)$ & $-4.70217+0.0097187 \mathrm{i}$ & $-4.70217+0.0097187 \mathrm{i}$ & $-4.70217+0.0097187 \mathrm{i}$ \\
\hline $\mathcal{G}_{1}\left(s_{1}=\frac{1}{2}-i, s_{2}=1+\frac{i}{3}\right)$ & $1.20737-4.67827 \mathrm{i}$ & $1.20735-4.67832 \mathrm{i}$ & $1.20736-4.67829 \mathrm{i}$ \\
\hline $\mathcal{G}_{2}\left(s_{1}=\frac{1}{22}, s_{2}=\frac{i}{13}\right)$ & $-3.25556+0.0100111 \mathrm{i}$ & $-3.25556+0.0100111 \mathrm{i}$ & $-3.25556+0.0100111 \mathrm{i}$ \\
\hline $\mathcal{G}_{2}\left(s_{1}=\frac{1}{2}-i, s_{2}=1+\frac{i}{3}\right)$ & $-2.60411-2.07626 \mathrm{i}$ & $-2.60411-2.07626 \mathrm{i}$ & $-2.60411-2.07626 \mathrm{i}$ \\
\hline
\end{tabular}

Table 4. Verification of the dispersion integral (2.8) against Lovelace-Shapiro model. We put $\operatorname{Disc} \mathcal{G}_{k}\left(s_{1}, s_{2}\right)$ from (A.5), (A.6), (A.7) in formula (2.8). For numerical purpose, we truncate the $k$-sum upto say $k_{\max }$.

with

$$
\lambda=\sqrt{\frac{4 a}{-a+2 k+1}+1}
$$

We put $\operatorname{Disc} \mathcal{G}_{k}\left(s_{1}, s_{2}\right)$ given in the above formulae in the dispersion relation (2.8). For numerical illustration, we truncate the $k$-sum at $k=k_{\max }$. We note that, because of $\delta\left(s_{1}-2 k-1\right)$, the $s_{1}^{\prime}$ integral can be easily done. The comparison is given the table below 4

\section{A.2 Inversion formulas and sum rules}

From the formulas (A.3), we can calculate the $s$-channel discontinuity $\mathcal{A}_{(L S)}^{(I)}\left(s_{1}, s_{2}\right)=$ $\operatorname{Disc}\left[\mathcal{M}_{(L S)}^{(I)}\left(s_{1}, s_{2}\right)\right]$.

$$
\begin{aligned}
& \mathcal{A}_{(L S)}^{(0)}\left(s_{1}, s_{2}\right)=\sum_{k=0}^{\infty} \pi \delta\left(s_{1}-2 k-1\right) \frac{3(-1)^{k}\left(-\frac{\Gamma\left(\frac{1}{2}-\frac{1}{4}(2 k+1)(\cos (\theta)-1)\right)}{\Gamma\left(-\frac{1}{4}(2 k+1)(\cos (\theta)+1)\right)}-\frac{\Gamma\left(\frac{1}{4}(2 \cos (\theta) k+2 k+\cos (\theta)+3)\right)}{\Gamma\left(\frac{1}{4}(2 k+1)(\cos (\theta)-1)\right)}\right)}{k !}, \\
& \mathcal{A}_{(L S)}^{(1)}\left(s_{1}, s_{2}\right)=\sum_{k=0}^{\infty} \pi \delta\left(s_{1}-2 k-1\right) \frac{2(-1)^{k}\left(\frac{\Gamma\left(\frac{1}{4}(2 \cos (\theta) k+2 k+\cos (\theta)+3)\right)}{\Gamma\left(\frac{1}{4}(2 k+1)(\cos (\theta)-1)\right)}-\frac{\Gamma\left(\frac{1}{2}-\frac{1}{4}(2 k+1)(\cos (\theta)-1)\right)}{\Gamma\left(-\frac{1}{4}(2 k+1)(\cos (\theta)+1)\right)}\right)}{k !} \\
& \mathcal{A}_{(L S)}^{(2)}\left(s_{1}, s_{2}\right)=0
\end{aligned}
$$

where $\cos (\theta)=1+\frac{2 s_{2}}{s_{1}}$. We will work in $d=4$ for illustration purpose. Now one can calculate the partial wave coefficients

$$
\Phi\left(s_{1} ; \alpha=1 / 2\right) a_{\ell}^{(I)}\left(s_{1}\right)=\frac{1}{2} \int_{-1}^{1} P_{\ell}(\cos (\theta)) \mathcal{A}_{(L S)}^{(I)}\left(s_{1}, s_{2}=s_{1} \frac{\cos (\theta)-1}{2}\right) d \cos (\theta) .
$$

We will truncate $\ell$-sum to $L_{\max }$ and $k$-sum to $k_{\max }$. Knowing the $a_{\ell}^{(I)}\left(s_{1}\right)$, we can calculate the $\mathcal{W}_{p, q}^{(k)}$ via formula $(2.16)$. Due to the $\delta\left(s_{1}-2 k-1\right)$, the $s_{1}$ integrals in $(2.16)$ can be easily done. Putting the equations (A.3) in (2.4), (2.5), (2.6) to get $\mathcal{G}_{k}\left(s_{1}, s_{2}\right)$, we can calculate the $\mathcal{W}_{p, q}^{(k)}$ directly after expanding the $\mathcal{G}_{k}\left(s_{1}, s_{2}\right)$ in powers of $x, y$. We can compare the directly calculated $\mathcal{W}_{p, q}^{(k)}$ with the inversion formula (2.16). The comparison is shown in the table 5 below

We can calculate $\mathcal{W}_{n-m, m}^{(k)}$ for $m>n$, the procedure is exactly same. Now since for $m>n$ which implies $(y / x)^{m} x^{n}$ i.e negative powers of $x$. There should not be any negative 


\begin{tabular}{|c|c|c|c|c|}
\hline $\mathcal{W}_{2,1}^{(\mathrm{k})}$ & Exact & $L_{\max }=6$ & $L_{\max }=10$ & $L_{\max }=20$ \\
\hline $\mathcal{W}_{2,1}^{(0)}$ & -2.49215 & -2.49217 & -2.49215 & -2.49215 \\
\hline $\mathcal{W}_{2,1}^{(1)}$ & -8.99964 & -8.99965 & -8.99964 & -8.99964 \\
\hline $\mathcal{W}_{2,1}^{(2)}$ & -6.00331 & -6.00331 & -6.00331 & -6.00331 \\
\hline
\end{tabular}

Table 5. $\mathcal{W}_{2,1}^{(k)}$ calculated directly using (A.3) in (2.4), (2.5), (2.6), compare with calculated using formula (2.16) and (A.10). We have truncated the $k$-sum to $k_{\max }=40$ and $\ell$-sum to $L_{\max }$.

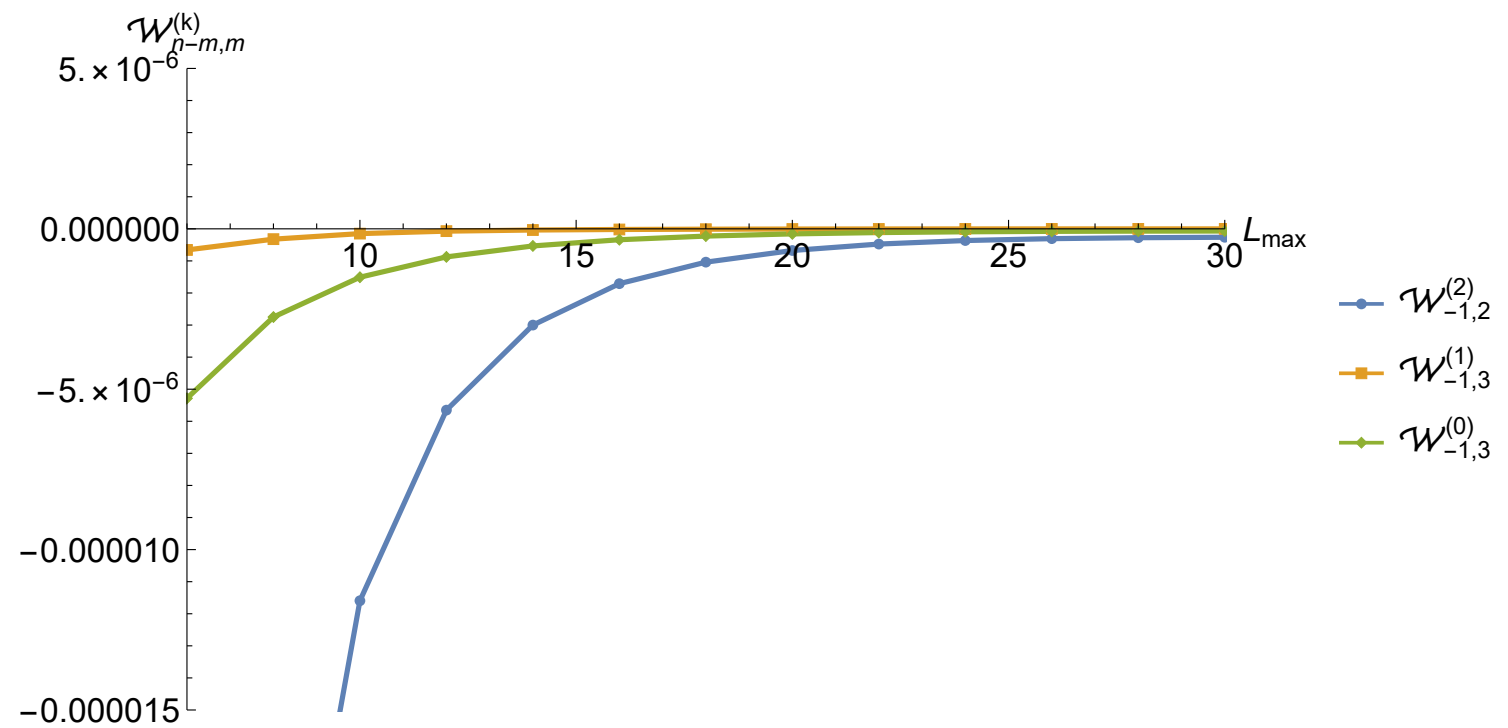

Figure 3. We calculate $\mathcal{W}_{n-m, m}^{(k)}$ for $m>n$, using formula $(2.16)$ and (A.10). The $\mathcal{W}_{n-m, m}^{(k)}$ for $m>n$ should vanish. We have truncated the $k$-sum to $k_{\max }=40$ and $\ell$-sum to $L_{\max }$. We see that as we increase $L_{\max }$ the $\mathcal{W}_{-1,3}^{(0)}, \mathcal{W}_{-1,3}^{(0)}, \mathcal{W}_{-1,2}^{(2)}$ go toward zero.

powers of $x$ as in the expression (2.14) due to locality. The coefficients $\mathcal{W}_{n-m, m}^{(k)}$ for $m>n$ should vanish. For Lovelace-Shapiro model, we have shown this in the figure 3 below

\section{B Positivity conditions on of $\mathcal{W}_{n, m}^{(1)}, \mathcal{W}_{n, m}^{(2)}$}

B.1 Finding $\Upsilon_{n, r, m}^{(1)}(\mu, \delta), \Upsilon_{n, r, m}^{(2)}(\mu, \delta), \chi_{n, r, m}^{(1)}(\mu, \delta), \chi_{n, r, m}^{(2)}(\mu, \delta)$

First, we take the combinations

$$
T_{1}=\sum_{r=0}^{m}\left(\Upsilon_{n, r, m}^{(1)}(\mu, \delta) \mathcal{W}_{n-r, r}^{(1)}+\bar{\chi}_{n, r, m}^{(1)}(\mu, \delta) \mathcal{W}_{n-r, r}^{(0)}\right)
$$

and

$$
T_{2}=\sum_{r=0}^{m}\left(\Upsilon_{n, r, m}^{(2)}(\mu, \delta) \mathcal{W}_{n-r, r}^{(2)}+\bar{\chi}_{n, r, m}^{(2)}(\mu, \delta) \mathcal{W}_{n-r, r}^{(0)}\right)
$$

From $T_{1}, T_{2}$ we collect all the coefficients of $a_{\ell}^{(I)}$ after we put the formula for $\mathcal{W}_{n-m, m}^{(k)}$ from the inversion formula (2.16). We will change the variable $s_{1}=\delta+\frac{2 \mu}{3}$. We always choose $\Upsilon_{n, m, m}^{(k)}(\mu, \delta)=1, \quad k=1,2$. 
For $T_{1}$, we collect the coefficients of $a_{\ell}^{(0)} C_{\ell-i}^{(\alpha+i)}\left(\frac{2 \mu}{3 \delta}+1\right), a_{\ell}^{(1)} C_{\ell-i}^{(\alpha+i)}\left(\frac{2 \mu}{3 \delta}+1\right)$ for $i=0,1,2,3, \ldots m-1$ and $a_{\ell}^{(2)} C_{\ell-m}^{(\alpha+m)}\left(\frac{2 \mu}{3 \delta}+1\right)$. Putting these coefficients to zero give solutions to $\Upsilon_{n, r, m}^{(1)}(\mu, \delta), \bar{\chi}_{n, r, m}^{(1)}(\mu, \delta)$. After that we put these solutions in $T_{1}$. Then we consider $\sum_{r=0}^{m-1} \hat{\chi}_{n, r, m}^{(1)}(\mu, \delta) \mathcal{W}_{n-r, r}^{(0)}+T_{1}$ and put the coefficients of $a_{\ell}^{(2)} C_{\ell-i}^{(\alpha+i)}\left(\frac{2 \mu}{3 \delta}+1\right)$ for $i=0,1,2,3, \ldots m-1$ to zero to get solutions for $\hat{\chi}_{n, r, m}^{(1)}(\mu, \delta)$. Now full solutions, we already have $\Upsilon_{n, r, m}^{(1)}(\mu, \delta)$, and $\chi_{n, r, m}^{(1)}(\mu, \delta)=\bar{\chi}_{n, r, m}^{(1)}(\mu, \delta)+\hat{\chi}_{n, r, m}^{(1)}(\mu, \delta)$. For example, we have worked out $m=1$ case

$$
\begin{aligned}
& \Upsilon_{n, 0,1} \mathcal{W}_{n, 0}^{(1)}+\mathcal{W}_{n, 1}^{(1)}+\bar{\chi}_{n, 0,1} \mathcal{W}_{n, 0}^{(0)}+\bar{\chi}_{n, 1,1} \mathcal{W}_{n, 1}^{(0)}, \\
& T_{1}= \frac{2 \bar{\chi}_{n, 0,1} C_{\ell}^{(\alpha)}\left(\frac{2 \mu}{3 \delta}+1\right)\left(a_{\ell}^{(0)}\left(s_{1}\right)+(N-1) a_{\ell}^{(2)}\left(s_{1}\right)\right)}{\pi N\left(\delta+\frac{2 \mu}{3}\right)^{2 n+1}} \\
&+\frac{3 \Upsilon_{n, 0,1} C_{\ell}^{(\alpha)}\left(\frac{2 \mu}{3 \delta}+1\right)\left(2 a_{\ell}^{(0)}\left(s_{1}\right)+N a_{\ell}^{(1)}\left(s_{1}\right)-(N+2) a_{\ell}^{(2)}\left(s_{1}\right)\right)}{2 \pi N\left(\delta+\frac{2 \mu}{3}\right)^{2(n+1)}} \\
&+\frac{\bar{\chi}_{n, 1,1}\left(a_{\ell}^{(0)}\left(s_{1}\right)+(N-1) a_{\ell}^{(2)}\left(s_{1}\right)\right)\left(8 \alpha(3 \delta+2 \mu) C_{\ell-1}^{(\alpha+1)}\left(\frac{2 \mu}{3 \delta}+1\right)-3 \delta(2 n+1) C_{\ell}^{(\alpha)}\left(\frac{2 \mu}{3 \delta}+1\right)\right)}{\pi \delta N 3\left(\delta+\frac{2 \mu}{3}\right)^{2(n+1)}} \\
&+\frac{\frac{12 \alpha\left(\delta+\frac{2 \mu}{3}\right) C_{\ell-1}^{(\alpha+1)}\left(\frac{2 \mu}{3 \delta}+1\right)\left(2 a_{\ell}^{(0)}\left(s_{1}\right)+N a_{\ell}^{(1)}\left(s_{1}\right)-(N+2) a_{\ell}^{(2)}\left(s_{1}\right)\right)}{\delta}}{2 \pi N\left(\delta+\frac{2 \mu}{3}\right)^{2 n+3}} \\
&-\frac{3 C_{\ell}^{(\alpha)}\left(\frac{2 \mu}{3 \delta}+1\right)\left(2 n a_{\ell}^{(0)}\left(s_{1}\right)+(n+4) N a_{\ell}^{(1)}\left(s_{1}\right)-n(N+2) a_{\ell}^{(2)}\left(s_{1}\right)\right)}{2 \pi N\left(\delta+\frac{2 \mu}{3}\right)^{2 n+3}} .
\end{aligned}
$$

Following the method described above, we get

$$
\Upsilon_{n, 0,1}=\frac{3(n+4)}{3 \delta+2 \mu}, \bar{\chi}_{n, 0,1}=\frac{27(2 n(N+2)-15 N+18)}{8(N-1)(3 \delta+2 \mu)^{2}}, \bar{\chi}_{n, 1,1}=\frac{9(N+2)}{4(N-1)(3 \delta+2 \mu)} .
$$

Now we add $\hat{\chi}_{n, 0,1}^{(1)}(\mu, \delta) \mathcal{W}_{n, 0}^{(0)}+T_{1}$ and put the coefficients of $a_{\ell}^{(2)} C_{\ell}^{(\alpha)}\left(\frac{2 \mu}{3 \delta}+1\right)$ to zero. We get

$$
\hat{\chi}_{n, 0,1}=\frac{81 N}{(N-1)(3 \delta+2 \mu)^{2}} .
$$

Then the full solution

$$
\chi_{n, 0,1}(\mu, \delta)=\frac{27(2 n+9)(N+2)}{8(N-1)(3 \delta+2 \mu)^{2}}, \Upsilon_{n, 0,1}(\mu, \delta)=\frac{3(n+4)}{3 \delta+2 \mu}, \chi_{n, 1,1}(\mu, \delta)=\frac{9(N+2)}{4(N-1)(3 \delta+2 \mu)} .
$$

Identical calculations can be repeated for higher values of $m$.

Similarly for $T_{2}$, we collect the coefficients of $a_{\ell}^{(2)} C_{\ell-i}^{(\alpha+i)}\left(\frac{2 \mu}{3 \delta}+1\right), a_{\ell}^{(1)} C_{\ell-i}^{(\alpha+i)}\left(\frac{2 \mu}{3 \delta}+1\right)$ for $i=0,1,2,3, \ldots m-1$ and $a_{\ell}^{(0)} C_{\ell-m}^{(\alpha+m)}\left(\frac{2 \mu}{3 \delta}+1\right)$. Putting these coefficients to zero give $\Upsilon_{n, r, m}^{(2)}(\mu, \delta), \bar{\chi}_{n, r, m}^{(2)}(\mu, \delta)$. Then we put these solutions in $T_{2}$. Now we consider $\sum_{r=0}^{m-1} \hat{\chi}_{n, r, m}^{(2)}(\mu, \delta) \mathcal{W}_{n-r, r}^{(0)}+T_{2}$ and put $a_{\ell}^{(0)} C_{\ell-i}^{(\alpha+i)}\left(\frac{2 \mu}{3 \delta}+1\right)$ for $i=0,1,2,3, \ldots m-1$ to zero to get solutions for $\hat{\chi}_{n, r, m}^{(2)}(\mu, \delta)$. Now full solutions, we already have $\Upsilon_{n, r, m}^{(2)}(\mu, \delta)$, and $\chi_{n, r, m}^{(2)}(\mu, \delta)=\bar{\chi}_{n, r, m}^{(2)}(\mu, \delta)+\hat{\chi}_{n, r, m}^{(2)}(\mu, \delta)$. 


\section{B.2 Proof of positivity conditions on of $\mathcal{W}_{n, m}^{(1)}, \mathcal{W}_{n, m}^{(2)}$}

After finding $\Upsilon_{n, r, m}^{(1)}(\mu, \delta), \Upsilon_{n, r, m}^{(2)}(\mu, \delta), \chi_{n, r, m}^{(1)}(\mu, \delta), \chi_{n, r, m}^{(2)}(\mu, \delta)$, one can check that coefficients of $a_{\ell}^{(I)} C_{\ell-i}^{(\alpha+i)}\left(\frac{2 \mu}{3 \delta}+1\right)$ for $i=0,1,2,3, \ldots m$ all are positive in i.e. (3.3), (3.4)

For example, lets consider (3.3) with $m=1$.

$$
\begin{aligned}
& \frac{\left(3\left(\delta-\delta_{0}\right)\left(3 \delta_{0}+2 \mu\right)((2 n+11) N+6)+3\left(\delta-\delta_{0}\right)^{2}(2 n+9)(N+2)+8 N\left(3 \delta_{0}+2 \mu\right)^{2}\right)}{4 \pi(N-1) N\left(\delta_{0}+\frac{2 \mu}{3}\right)^{2}\left(\delta+\frac{2 \mu}{3}\right)^{2 n+3}} a_{\ell}^{(0)} C_{\ell}^{(\alpha)}\left(\frac{2 \mu}{3 \delta}+1\right) \\
& +\frac{2 \alpha 9^{n+2}(3 \delta+2 \mu)^{-2(n+1)}\left(N\left(3 \delta_{0}+2 \mu\right)+\left(\delta-\delta_{0}\right)(N+2)\right)}{\pi \delta(N-1) N\left(3 \delta_{0}+2 \mu\right)} a_{\ell}^{(0)} C_{\ell-1}^{(\alpha+1)}\left(\frac{2 \mu}{3 \delta}+1\right) \\
& \times \frac{\left(\delta-\delta_{0}\right) 3^{2 n+5}(n+4)(3 \delta+2 \mu)^{-2 n-3}}{2 \pi\left(3 \delta_{0}+2 \mu\right)} a_{\ell}^{(1)} C_{\ell}^{(\alpha)}\left(\frac{2 \mu}{3 \delta}+1\right)+\frac{6 \alpha\left(\delta+\frac{2 \mu}{3}\right)^{-2(n+1)}}{\pi \delta} a_{\ell}^{(1)} C_{\ell-1}^{(\alpha+1)}\left(\frac{2 \mu}{3 \delta}+1\right) \\
& \times \frac{\left(\delta-\delta_{0}\right) 9^{n+3}(N+2)(3 \delta+2 \mu)^{-2 n-3}\left(9 \delta_{0}+6 \mu+\left(\delta-\delta_{0}\right)(2 n+9)\right)}{4 \pi N\left(3 \delta_{0}+2 \mu\right)^{2}} a_{\ell}^{(2)} C_{\ell}^{(\alpha)}\left(\frac{2 \mu}{3 \delta}+1\right) \\
& +\frac{2 \alpha\left(\delta-\delta_{0}\right) 9^{n+2}(N+2)(3 \delta+2 \mu)^{-2(n+1)}}{\pi \delta N\left(3 \delta_{0}+2 \mu\right)} a_{\ell}^{(2)} C_{\ell-1}^{(\alpha+1)}\left(\frac{2 \mu}{3 \delta}+1\right) \geq 0 .
\end{aligned}
$$

The above is always positive for $\delta \geq \delta_{0} \geq 0, \alpha \geq 0, \mu \geq 0$. Similarly one can check for any $m$ that equation (3.3) from the fact that coefficients of $a_{\ell}^{(I)} C_{\ell-i}^{(\alpha+i)}\left(\frac{2 \mu}{3 \delta}+1\right)$ for $i=0,1,2,3, \ldots m$ are all positive.

Similar arguments holds for (3.4). The coefficients of $a_{\ell}^{(0)} C_{\ell-i}^{(\alpha+i)}\left(\frac{2 \mu}{3 \delta}+1\right)$ for $i=$ $0,1,2,3, \ldots m$ and coefficients of $a_{\ell}^{(2)} C_{\ell-i}^{(\alpha+i)}\left(\frac{2 \mu}{3 \delta}+1\right)$ for $i=0,1,2,3, \ldots m$ are always positive. But for coefficients of $a_{\ell}^{(1)}$, we can check numerically that they are indeed positive (i.e. the total sum of the all Gegenbauer polynomials, not individual coefficients of $C_{\ell-i}^{(\alpha+i)}\left(\frac{2 \mu}{3 \delta}+1\right)$ for $\left.i=0,1,2,3, \ldots m\right)$.

\section{Positivity of Disc $\left[\left(1-\frac{2 \delta_{2, k}}{3}\right) \mathcal{G}_{0}+\delta_{1, k} \mathcal{G}_{1}+\delta_{2, k} \mathcal{G}_{2}\right]\left(s_{1}^{\prime} ; s_{2}^{(+)}\left(s_{1}^{\prime}, a\right)\right)$}

In this section, we prove that Disc $\left[\left(1-\frac{2 \delta_{2, k}}{3}\right) \mathcal{G}_{0}+\delta_{1, k} \mathcal{G}_{1}+\delta_{2, k} \mathcal{G}_{2}\right]\left(s_{1}^{\prime} ; s_{2}^{(+)}\left(s_{1}^{\prime}, a\right)\right)>0$ for $s_{1}^{\prime} \geq \frac{2 \mu}{3}, N \geq 3, \mu \geq 4$ and range of $a$ in case of $k=0$ is given by $-\frac{2 \mu}{9}<a<\frac{2 \mu}{3}$, while in case of $k=1,2$ range of $a$ is given by $-\frac{2 \mu}{9}<a<\frac{2 \mu}{9}$. We will be using the positivity of the absorptive part of isospin amplitudes, namely $\mathcal{A}^{(I)}\left(s_{1}^{\prime} ; s_{2}^{(+)}\left(s_{1}^{\prime}, a\right)\right)$ is non-negative for $-\frac{2 \mu}{9}<a<\frac{2 \mu}{3}, s_{1}^{\prime} \geq \frac{2 \mu}{3}$. This follows from the partial wave expansion (2.11), (2.12) and the positivity of the Gegenbauer polynomials,

$$
C_{\ell}^{(\alpha)}\left(\sqrt{\xi\left(s_{1}, a\right)}\right)>0 \text { if }-\frac{2 \mu}{9}<a<\frac{2 \mu}{3}, s_{1}^{\prime} \geq \frac{2 \mu}{3} .
$$

This is true because $\sqrt{\xi\left(s_{1}, a\right)}>1$ for $-\frac{2 \mu}{9}<a<\frac{2 \mu}{3}, s_{1}^{\prime} \geq \frac{2 \mu}{3}$.

$\boldsymbol{k}=\mathbf{0}$ case. For $k=0$, we have

$$
\operatorname{Disc} \mathcal{G}_{0}\left(s_{1}^{\prime} ; s_{2}^{(+)}\left(s_{1}^{\prime}, a\right)\right)=\frac{\mathcal{A}^{(0)}\left(s_{1}^{\prime} ; s_{2}^{(+)}\left(s_{1}^{\prime}, a\right)\right)+(N-1) \mathcal{A}^{(2)}\left(s_{1}^{\prime} ; s_{2}^{(+)}\left(s_{1}^{\prime}, a\right)\right)}{N} .
$$

Therefore from non-negativity of $\mathcal{A}^{(I)}\left(s_{1}^{\prime} ; s_{2}^{(+)}\left(s_{1}^{\prime}, a\right)\right)$, we find that $\operatorname{Disc} \mathcal{G}_{0}\left(s_{1}^{\prime} ; s_{2}^{(+)}\left(s_{1}^{\prime}, a\right)\right)$ is non-negative for $-\frac{2 \mu}{9}<a<\frac{2 \mu}{3}, s_{1}^{\prime} \geq \frac{2 \mu}{3}$. 
$\boldsymbol{k}=\mathbf{1}$ case. For $k=1$, we have

$$
\begin{aligned}
& \operatorname{Disc}\left[\mathcal{G}_{0}+\mathcal{G}_{1}\right]\left(s_{1}^{\prime} ; s_{2}^{(+)}\left(s_{1}^{\prime}, a\right)\right)=\left[\frac{\frac{1}{2 s_{1}^{\prime}-3 a}+\frac{1}{s_{1}^{\prime}}+1}{N}\right] \mathcal{A}^{(0)}\left(s_{1}^{\prime} ; s_{2}^{(+)}\left(s_{1}^{\prime}, a\right)\right) \\
& +\left[\frac{3\left(3 a-s_{1}^{\prime}\right) \sqrt{1-\frac{4 a}{3 a+s_{1}^{\prime}}}}{2 s_{1}^{\prime}\left(3 a-2 s_{1}^{\prime}\right)}\right] \mathcal{A}^{(1)}\left(s_{1}^{\prime} ; s_{2}^{(+)}\left(s_{1}^{\prime}, a\right)\right)+\left[\frac{\frac{N+2}{3 a-2 s_{1}^{\prime}}-\frac{N+2}{s_{1}^{\prime}}+2 N-2}{2 N}\right] \mathcal{A}^{(2)}\left(s_{1}^{\prime} ; s_{2}^{(+)}\left(s_{1}^{\prime}, a\right)\right) .
\end{aligned}
$$

$\boldsymbol{k}=\mathbf{2}$ case. For $k=2$ we have

$$
\begin{aligned}
& \operatorname{Disc}\left[\frac{\mathcal{G}_{0}}{3}+\mathcal{G}_{2}\right]\left(s_{1}^{\prime} ; s_{2}^{(+)}\left(s_{1}^{\prime}, a\right)\right)=\left[-\frac{-3 a\left(s_{1}^{\prime}\right)^{2}+9 a+2\left(s_{1}^{\prime}\right)^{3}-9 s_{1}^{\prime}}{9 a N\left(s_{1}^{\prime}\right)^{2}-6 N\left(s_{1}^{\prime}\right)^{3}}\right] \mathcal{A}^{(0)}\left(s_{1}^{\prime} ; s_{2}^{(+)}\left(s_{1}^{\prime}, a\right)\right) \\
& +\left[\frac{9\left(s_{1}^{\prime}-a\right)^{3 / 2}}{2\left(s_{1}^{\prime}\right)^{2} \sqrt{3 a+s_{1}^{\prime}}\left(2 s_{1}^{\prime}-3 a\right)}\right] \mathcal{A}^{(1)}\left(s_{1}^{\prime} ; s_{2}^{(+)}\left(s_{1}^{\prime}, a\right)\right)+\left[\frac{\frac{3(N+2)}{2 s_{1}^{\prime}-3 a}+\frac{3(N+2)}{s_{1}^{\prime}}+2(N-1) s_{1}^{\prime}}{6 N s_{1}^{\prime}}\right] \mathcal{A}^{(2)}\left(s_{1}^{\prime} ; s_{2}^{(+)}\left(s_{1}^{\prime}, a\right)\right) .
\end{aligned}
$$

In both of the cases $k=1,2$, one can see that each coefficients of $\mathcal{A}^{(I)}\left(s_{1}^{\prime} ; s_{2}^{(+)}\left(s_{1}^{\prime}, a\right)\right)$ are positive if we consider the range $-\frac{2 \mu}{9}<a<\frac{2 \mu}{9}, s_{1}^{\prime} \geq \frac{2 \mu}{3}, N \geq 3, \mu \geq 4$. Once we see all coefficients are positive. Then using the non-negativity of the $\mathcal{A}^{(I)}\left(s_{1}^{\prime} ; s_{2}^{(+)}\left(s_{1}^{\prime}, a\right)\right)$. We conclude that $k=1,2$ cases are non-negativity for $s_{1}^{\prime} \geq \frac{2 \mu}{3}, N \geq 3, \mu \geq 4$ and range of $a$ is given by $-\frac{2 \mu}{9}<a<\frac{2 \mu}{9}$.

\section{Verifications of bounds with known theories}

\section{D.1 The 2-loop chiral perturbation theory}

In the 2-loop chiral perturbation theory, we use the experimental values for the parameters in the amplitude (we use the amplitude given in [22, 62]).

$$
\begin{array}{lll}
b_{1}=-0.0785239, & b_{2}=0.0747244, & b_{3}=-0.00208975, \\
b_{4}=0.0046861, & b_{5}=0.000143563, & b_{6}=0.0000942385
\end{array}
$$

We find the coefficients

$$
\begin{array}{lllll}
\widehat{\mathcal{W}}_{0,1}^{(0)}=-0.16534, & \frac{\widehat{\mathcal{W}}_{0,2}^{(0)}}{\widehat{\mathcal{W}}_{1,0}^{(0)}}=0.00156937, & \frac{\widehat{\mathcal{W}}_{1,1}^{(0)}}{\widehat{\mathcal{W}}_{1,0}^{(0)}}=-0.0102986, & \frac{\widehat{\mathcal{W}}_{2,0}^{(0)}}{\widehat{\mathcal{W}}_{1,0}^{(0)}}=0.0182756, & \frac{\widehat{\mathcal{W}}_{3,0}^{(0)}}{\widehat{\mathcal{W}}_{1,0}^{(0)}}=0.00104625, \\
\widehat{\mathcal{W}}_{0,1}^{(1)}=-0.164701, & \frac{\widehat{\mathcal{W}}_{0,2}^{(1)}}{\widehat{\mathcal{W}}_{1,0}^{(1)}}=0.00166561, & \frac{\widehat{\mathcal{W}}_{1,1}^{(1)}}{\widehat{\mathcal{W}}_{1,0}^{(1)}}=-0.0111136, & \frac{\widehat{\mathcal{W}}_{2,0}^{(1)}}{\widehat{\mathcal{W}}_{1,0}^{(1)}}=0.0200637, & \frac{\widehat{\mathcal{W}}_{3,0}^{(1)}}{\widehat{\mathcal{W}}_{1,0}^{(1)}}=0.00119862, \\
\widehat{\mathcal{W}}_{1,0}^{(1)}=- & \widehat{\mathcal{W}}_{0,2}^{(2)}=0.0137037, & \frac{\widehat{\mathcal{W}}_{1,1}^{(2)}}{\widehat{\mathcal{W}}_{1,0}^{(2)}}=-0.00892794, & \frac{\widehat{\mathcal{W}}_{2,0}^{(2)}}{\widehat{\mathcal{W}}_{1,0}^{(2)}}=0.0158325, & \frac{\widehat{\mathcal{W}}_{3,0}^{(2)}}{\widehat{\mathcal{W}}_{1,0}^{(2)}}=0.000818889 . \\
\widehat{\mathcal{W}}_{0,1}^{(2)}=-0.161194, & \frac{\widehat{\mathcal{W}}_{1,0}^{(2)}}{\widehat{\mathcal{W}}_{1,0}^{(2)}}=-0.0
\end{array}
$$

We can easily see that all of these coefficients satisfy the bounds listed in table 1,2 . We can compute the $\mathcal{C}_{p, q}$ using the equation (5.10). Some of them are

$$
\begin{array}{llll}
\mathcal{C}_{0,4}=-0.0679822, & \mathcal{C}_{0,5}=-0.000596033, & \mathcal{C}_{0,6}=-0.000733546, & \mathcal{C}_{0,7}=-0.0000507897, \\
\mathcal{C}_{1,1}=0.0551134, & \mathcal{C}_{1,2}=0.0864709, & \mathcal{C}_{1,3}=-0.0095459, & \mathcal{C}_{1,4}=0.00077458, \mathcal{C}_{2,0}=0.15424, \\
\mathcal{C}_{2,1}=0.0264102, & \mathcal{C}_{2,2}=0.00454459, & \mathcal{C}_{3,0}=0.00251334 .
\end{array}
$$

Note that in equation (5.10) we have used values in equations (D.2) with our normalizations $\widehat{\mathcal{W}}_{1,0}^{(k)}=1$. All of these $\mathcal{C}_{p, q}$ satisfy the two sided bounds presented in table 3 . 


\section{D.2 The $O(3)$ Lovelace-Shapiro model}

We demonstrate our bounds are satisfied by the $O(3)$ Lovelace-Shapiro model eq (A.1) with $\beta_{0}=\beta^{\prime}=1 / 2$. Using the isospin amplitudes of the Lovelace-Shapiro model (A.3), one can compute the $\widehat{\mathcal{W}}_{p, q}^{(k)}$. Our bounds assumed the lower limit of the $s_{1}^{\prime}$ integral as $\frac{8}{3}$ (for $\mu=4$ ), while for the $O(3)$ Lovelace-Shapiro model lower limit is $s_{1}^{\prime}=1$. Therefore, in order to compare with the bounds given in 2 , we need to multiply with appropriate powers of $\frac{3}{8}$. In order to match with the conventions of the EFT scale, we multiply $\frac{\widehat{\mathcal{W}}_{n-m, m}^{(k)}}{\widehat{\mathcal{W}}_{1,0}^{(k)}}$ by $\left(\frac{3}{8}\right)^{m+2 n-2}($ see $[3,23])$

$$
\begin{array}{lll}
\frac{3 \widehat{\mathcal{W}}_{0,1}^{(0)}}{8 \widehat{\mathcal{W}}_{1,0}^{(0)}}=-0.472497, & \frac{9 \widehat{\mathcal{W}}_{2,0}^{(0)}}{64 \widehat{\mathcal{W}}_{1,0}^{(0)}}=0.130058, & \frac{27 \widehat{\mathcal{W}}_{1,1}^{(0)}}{512 \widehat{\mathcal{W}}_{1,0}^{(0)}}=-0.120704, \\
\frac{3 \widehat{\mathcal{W}}_{0,1}^{(1)}}{8 \widehat{\mathcal{W}}_{1,0}^{(1)}}=-0.661605, & \frac{9 \widehat{\mathcal{W}}_{2,0}^{(1)}}{64 \widehat{\mathcal{W}}_{1,0}^{(1)}}=0.135717, & \frac{27 \widehat{\mathcal{W}}_{1,1}^{(1)}}{512 \widehat{\mathcal{W}}_{1,0}^{(1)}}=-0.145721, \\
\frac{3 \widehat{\mathcal{W}}_{0,1}^{(2)}}{8 \widehat{\mathcal{W}}_{1,0}^{(2)}}=-0.385458, & \frac{9 \widehat{\mathcal{W}}_{2,0}^{(2)}}{64 \widehat{\mathcal{W}}_{1,0}^{(2)}}=0.13871, & \frac{27 \widehat{\mathcal{W}}_{1,1}^{(2)}}{512 \widehat{\mathcal{W}}_{1,0}^{(2)}}=-0.106502
\end{array}
$$

We can easily see that the r.h.s. of the above equation all satisfy the two sided bounds presented in table 1, 2. Using the above equation and replacing $\frac{\widehat{\mathcal{W}}_{n-m, m}^{(k)}}{\widehat{\mathcal{W}}_{1,0}^{(k)}} \rightarrow\left(\frac{3}{8}\right)^{m+2 n-2} \frac{\widehat{\mathcal{W}}_{n-m, m}^{(k)}}{\widehat{\mathcal{W}}_{1,0}^{(k)}}$, we can calculate $\mathcal{C}_{p, q}$ from (5.10). With our normalization $\widehat{\mathcal{W}}_{1,0}^{(k)}=1$, we find

$$
\begin{array}{llll}
\mathcal{C}_{0,4}=-0.0307213, & \mathcal{C}_{0,5}=-0.00188633, & \mathcal{C}_{1,1}=0.157499, & \mathcal{C}_{1,2}=0.0977439, \\
\mathcal{C}_{1,3}=0.0111333, & \mathcal{C}_{2,0}=0.191501, & \mathcal{C}_{2,1}=0.0890061
\end{array}
$$

Notice that these $\mathcal{C}_{p, q}$ satisfy the inequalities listed in table 3.

Open Access. This article is distributed under the terms of the Creative Commons Attribution License (CC-BY 4.0), which permits any use, distribution and reproduction in any medium, provided the original author(s) and source are credited.

\section{References}

[1] W. Rogosinski, Über positive harmonische Entwicklungen and typisch-reelle Potenzreihen, Math. Z. 35 (1932) 93.

[2] Y. Komatu, The coefficients of typically-real Laurent series, Kodai Math. Sem. Rep. 9 (1957) 42.

[3] P. Raman and A. Sinha, QFT, EFT and GFT, arXiv:2107.06559 [INSPIRE].

[4] P. Haldar, A. Sinha and A. Zahed, Quantum field theory and the Bieberbach conjecture, SciPost Phys. 11 (2021) 002 [arXiv:2103.12108] [INSPIRE].

[5] G. Auberson and N.N. Khuri, Rigorous parametric dispersion representation with three-channel symmetry, Phys. Rev. D 6 (1972) 2953 [INSPIRE]. 
[6] A. Sinha and A. Zahed, Crossing symmetric dispersion relations in quantum field theories, Phys. Rev. Lett. 126 (2021) 181601 [arXiv:2012.04877] [InSPIRE].

[7] S. Mandelstam, Determination of the pion-nucleon scattering amplitude from dispersion relations and unitarity. General theory, Phys. Rev. 112 (1958) 1344 [InSPIRE].

[8] H.M. Nussenzveig, Causality and dispersion relations, Academic Press, (1972).

[9] A. Martin, Scattering theory: unitarity, analyticity and crossing, Lect. Notes Phys. 3 (1969) 1 [INSPIRE].

[10] M. Froissart, Asymptotic behavior and subtractions in the Mandelstam representation, Phys. Rev. 123 (1961) 1053 [INSPIRE].

[11] P. Haldar and A. Sinha, Froissart bound for/from CFT Mellin amplitudes, SciPost Phys. 8 (2020) 095 [arXiv: 1911.05974] [InSPIRE].

[12] D. Meltzer, Dispersion formulas in QFTs, CFTs, and holography, JHEP 05 (2021) 098 [arXiv:2103.15839] [INSPIRE].

[13] D. Meltzer, The inflationary wavefunction from analyticity and factorization, arXiv: 2107.10266 [INSPIRE].

[14] A. Adams, N. Arkani-Hamed, S. Dubovsky, A. Nicolis and R. Rattazzi, Causality, analyticity and an IR obstruction to UV completion, JHEP 10 (2006) 014 [hep-th/0602178] [INSPIRE].

[15] J. Penedones, J.A. Silva and A. Zhiboedov, Nonperturbative Mellin amplitudes: existence, properties, applications, JHEP 08 (2020) 031 [arXiv:1912.11100] [INSPIRE].

[16] D. Carmi, J. Penedones, J.A. Silva and A. Zhiboedov, Applications of dispersive sum rules: є-expansion and holography, SciPost Phys. 10 (2021) 145 [arXiv: 2009.13506] [INSPIRE].

[17] S. Caron-Huot, D. Mazac, L. Rastelli and D. Simmons-Duffin, Dispersive CFT sum rules, JHEP 05 (2021) 243 [arXiv: 2008. 04931] [INSPIRE].

[18] R. Gopakumar, A. Sinha and A. Zahed, Crossing symmetric dispersion relations for Mellin amplitudes, Phys. Rev. Lett. 126 (2021) 211602 [arXiv:2101.09017] [INSPIRE].

[19] C. de Rham, S. Melville, A.J. Tolley and S.-Y. Zhou, Positivity bounds for scalar field theories, Phys. Rev. D 96 (2017) 081702 [arXiv:1702.06134] [INSPIRE].

[20] B. Bellazzini, J. Elias Miró, R. Rattazzi, M. Riembau and F. Riva, Positive moments for scattering amplitudes, Phys. Rev. D 104 (2021) 036006 [arXiv:2011.00037] [INSPIRE].

[21] A.J. Tolley, Z.-Y. Wang and S.-Y. Zhou, New positivity bounds from full crossing symmetry, JHEP 05 (2021) 255 [arXiv: 2011. 02400] [INSPIRE].

[22] Y.-J. Wang, F.-K. Guo, C. Zhang and S.-Y. Zhou, Generalized positivity bounds on chiral perturbation theory, JHEP 07 (2020) 214 [arXiv: 2004.03992] [INSPIRE].

[23] S. Caron-Huot and V. Van Duong, Extremal effective field theories, JHEP 05 (2021) 280 [arXiv: 2011.02957] [INSPIRE].

[24] S. Caron-Huot, D. Mazac, L. Rastelli and D. Simmons-Duffin, Sharp boundaries for the swampland, JHEP 07 (2021) 110 [arXiv:2102.08951] [INSPIRE].

[25] S. Caron-Huot, D. Mazac, L. Rastelli and D. Simmons-Duffin, AdS bulk locality from sharp CFT bounds, JHEP 11 (2021) 164 [arXiv:2106.10274] [INSPIRE].

[26] N. Arkani-Hamed and Y.-T. Huang, Lectures at the CERN winter school on supergravity, strings and gauge theory. 
[27] M.B. Green and C. Wen, Superstring amplitudes, unitarily, and Hankel determinants of multiple zeta values, JHEP 11 (2019) 079 [arXiv: 1908.08426] [INSPIRE].

[28] N. Arkani-Hamed, T.-C. Huang and Y.-T. Huang, The EFT-hedron, JHEP 05 (2021) 259 [arXiv: 2012.15849] [INSPIRE].

[29] L.-Y. Chiang, Y.-T. Huang, W. Li, L. Rodina and H.-C. Weng, Into the EFT-hedron and UV constraints from IR consistency, arXiv:2105.02862 [INSPIRE].

[30] Z. Bern, D. Kosmopoulos and A. Zhiboedov, Gravitational effective field theory islands, low-spin dominance, and the four-graviton amplitude, J. Phys. A 54 (2021) 344002 [arXiv:2103.12728] [INSPIRE].

[31] A.-C. Davis and S. Melville, Scalar fields near compact objects: resummation versus UV completion, JCAP 11 (2021) 012 [arXiv:2107.00010] [INSPIRE].

[32] G. Mahoux, S.M. Roy and G. Wanders, Physical pion pion partial-wave equations based on three channel crossing symmetry, Nucl. Phys. B 70 (1974) 297 [InSPIRE].

[33] R. Roskies, Crossing restrictions on $\pi \pi$ partial waves, Nuovo Cim. A 65 (1970) 467 [INSPIRE].

[34] B. Ananthanarayan, The low-energy expansion for pion pion scattering and crossing symmetry in dispersion relations, Phys. Rev. D 58 (1998) 036002 [hep-ph/9802338] [INSPIRE].

[35] M.S. Robertson, On coefficients of a typically real function, Bull. Amer. Math. Soc. 41 (1935) 565.

[36] A. Bose, A. Sinha and S.S. Tiwari, Selection rules for the S-matrix bootstrap, SciPost Phys. 10 (2021) 122 [arXiv:2011.07944] [INSPIRE].

[37] A. Bose, P. Haldar, A. Sinha, P. Sinha and S.S. Tiwari, Relative entropy in scattering and the S-matrix bootstrap, SciPost Phys. 9 (2020) 081 [arXiv: 2006.12213] [INSPIRE].

[38] A.M. Polyakov, Nonhamiltonian approach to conformal quantum field theory, Zh. Eksp. Teor. Fiz. 66 (1974) 23 [Sov. Phys. JETP 39 (1974) 9] [INSPIRE].

[39] K. Sen and A. Sinha, On critical exponents without Feynman diagrams, J. Phys. A 49 (2016) 445401 [arXiv: 1510.07770] [INSPIRE].

[40] R. Gopakumar, A. Kaviraj, K. Sen and A. Sinha, Conformal bootstrap in Mellin space, Phys. Rev. Lett. 118 (2017) 081601 [arXiv:1609.00572] [INSPIRE].

[41] R. Gopakumar, A. Kaviraj, K. Sen and A. Sinha, A Mellin space approach to the conformal bootstrap, JHEP 05 (2017) 027 [arXiv: 1611.08407] [INSPIRE].

[42] R. Gopakumar and A. Sinha, On the Polyakov-Mellin bootstrap, JHEP 12 (2018) 040 [arXiv: 1809.10975] [INSPIRE].

[43] P. Dey, A. Kaviraj and A. Sinha, Mellin space bootstrap for global symmetry, JHEP 07 (2017) 019 [arXiv: 1612.05032] [INSPIRE].

[44] P. Ferrero, K. Ghosh, A. Sinha and A. Zahed, Crossing symmetry, transcendentality and the Regge behaviour of $1 d$ CFTs, JHEP 07 (2020) 170 [arXiv:1911.12388] [INSPIRE].

[45] K. Ghosh and A. Zahed, Witten block expansion of CFT Mellin amplitudes with $O(N)$ symmetry, work in progress. 
[46] A. Kaviraj, Crossing antisymmetric Polyakov blocks + dispersion relation, arXiv: 2109.02658 [INSPIRE].

[47] K. Ghosh, A. Kaviraj and M.F. Paulos, Charging up the functional bootstrap, JHEP 10 (2021) 116 [arXiv: 2107.00041] [INSPIRE].

[48] S. Kundu, Swampland conditions for higher derivative couplings from CFT, arXiv:2104.11238 [INSPIRE].

[49] S. Caron-Huot, D. Mazac, L. Rastelli and D. Simmons-Duffin, AdS bulk locality from sharp CFT bounds, JHEP 11 (2021) 164 [arXiv:2106.10274] [INSPIRE].

[50] M.F. Paulos, Dispersion relations and exact bounds on CFT correlators, JHEP 08 (2021) 166 [arXiv: 2012.10454] [INSPIRE].

[51] N. Arkani-Hamed, Y.-T. Huang and S.-H. Shao, On the positive geometry of conformal field theory, JHEP 06 (2019) 124 [arXiv: 1812.07739] [InSPIRE].

[52] K. Sen, A. Sinha and A. Zahed, Positive geometry in the diagonal limit of the conformal bootstrap, JHEP 11 (2019) 059 [arXiv: 1906.07202] [INSPIRE].

[53] Y.-T. Huang, W. Li and G.-L. Lin, The geometry of optimal functionals, arXiv:1912.01273 [INSPIRE].

[54] A.L. Guerrieri, J. Penedones and P. Vieira, Bootstrapping QCD using pion scattering amplitudes, Phys. Rev. Lett. 122 (2019) 241604 [arXiv:1810.12849] [INSPIRE].

[55] A.L. Guerrieri, A. Homrich and P. Vieira, Dual S-matrix bootstrap. Part I. $2 D$ theory, JHEP 11 (2020) 084 [arXiv:2008.02770] [INSPIRE].

[56] Y. He and M. Kruczenski, S-matrix bootstrap in $3+1$ dimensions: regularization and dual convex problem, JHEP 08 (2021) 125 [arXiv:2103.11484] [INSPIRE].

[57] J. Elias Miró and A. Guerrieri, Dual EFT bootstrap: QCD flux tubes, JHEP 10 (2021) 126 [arXiv: 2106.07957] [INSPIRE].

[58] A. Guerrieri and A. Sever, Rigorous bounds on the analytic S-matrix, arXiv:2106.10257 [INSPIRE].

[59] C. Lovelace, A novel application of Regge trajectories, Phys. Lett. B 28 (1968) 264 [INSPIRE].

[60] J.A. Shapiro, Narrow-resonance model with Regge behavior for $\pi \pi$ scattering, Phys. Rev. 179 (1969) 1345 [INSPIRE].

[61] M. Bianchi, D. Consoli and P. Di Vecchia, On the N-pion extension of the Lovelace-Shapiro model, JHEP 03 (2021) 119 [arXiv: 2002.05419] [INSPIRE].

[62] J. Bijnens, G. Colangelo, G. Ecker, J. Gasser and M.E. Sainio, Elastic $\pi \pi$ scattering to two loops, Phys. Lett. B 374 (1996) 210 [hep-ph/9511397] [InSPIRE]. 